\title{
Essential Role of Sptan 1 in Cochlear Hair Cell Morphology and Function Via Focal Adhesion Signaling
}

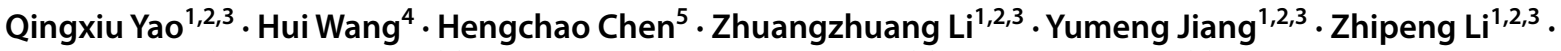 \\ Jiping Wang ${ }^{1,2,3} \cdot$ Yazhi Xing ${ }^{1,2,3} \cdot$ Feng Liu ${ }^{1,2,3} \cdot$ Dongzhen $\mathrm{Yu}^{1,2,3}$ (1) Shankai Yin ${ }^{1,2,3}$
}

Received: 10 June 2021 / Accepted: 31 August 2021 / Published online: 27 October 2021

(c) The Author(s) 2021

\begin{abstract}
Hearing loss is the most common human sensory deficit. Hearing relies on stereocilia, inserted into the cuticular plate of hair cells (HCs), where they play an important role in the perception of sound and its transmission. Although numerous genes have been associated with hearing loss, the function of many hair cell genes has yet to be elucidated. Herein, we focused on nonerythroid spectrin $\alpha$ II (SPTAN1), abundant in the cuticular plate, surrounding the rootlets of stereocilia and along the plasma membrane. Interestingly, mice with HC-specific Sptan 1 knockout exhibited rapid deafness, abnormal formation of stereocilia and cuticular plates, and loss of $\mathrm{HCs}$ from middle and apical turns of the cochlea during early postnatal stages. Additionally, Sptanl deficiency led to the decreased spreading of House Ear Institute-Organ of Corti 1 cells, and induced abnormal formation of focal adhesions and integrin signaling in mouse HCs. Altogether, our findings highlight SPTAN1 as a critical molecule for HC stereocilia morphology and auditory function via regulation of focal adhesion signaling.
\end{abstract}

Keywords Sptan $1 \cdot \alpha$ II spectrin $\cdot$ Deafness $\cdot$ Stereocilia $\cdot$ Hair cell

\section{Introduction}

Hearing loss is the most common sensory disorder worldwide with genetic factors contributing to approximately half of congenital hearing loss cases [1]. Sensory epithelial hair

Qingxiu Yao, Hui Wang, and Hengchao Chen contributed equally to this work.

Feng Liu

liufeng@sibs.ac.cn

$\triangle$ Dongzhen Yu

drdzyu@126.com

1 Department of Otolaryngology-Head and Neck Surgery, Shanghai Jiao Tong University Affiliated Sixth People's Hospital, Shanghai 200233, China

2 Otolaryngology Institute of Shanghai Jiao Tong University, Shanghai 200233, China

3 Shanghai Key Laboratory of Sleep Disordered Breathing, Shanghai 200233, China

4 ENT Institute and Otorhinolaryngology Department, Affiliated Eye and ENT Hospital, Fudan University, Shanghai 200031, China

5 The First Affiliated Hospital, Zhejiang University School of Medicine, Hangzhou 310003, China cells, including the inner hair cells (IHCs) and outer hair cells (OHCs), contribute to normal hearing and balance via the conversion of movements initiated by sound waves into electrical signals [2-4]. Stereocilia, located on the top of hair cells (HCs), are microvillus-like protrusions containing actin filaments (F-actin) [5-7]. The rootlets of stereocilia insert into the dense F-actin mesh known as the cuticular plate, speculated to strengthen and provide additional anchoring for the stereocilia [7-9]. Previous studies have shown that the cuticular plate is enriched in various proteins, including myosin VI, which play important roles in the development and/or maintenance of stereocilia [10-16]. In our previous study, we found that myosin VI interacted with nonerythroid $\alpha$ II-spectrin (SPTAN1) [17], also known as alpha fodrin, encoded by Sptan1. Of note, SPTAN1 is distributed in the cuticular plate and along the lateral wall in HCs [18]; therefore, we speculated that Sptan1 may play a critical role in HCs.

SPTAN1 is an essential cytoskeletal protein that ensures vital cellular properties, including polarity and cell stabilization. In addition, it is involved in cell adhesion, intercellular contact, and apoptosis [19]. Previous studies have demonstrated that Sptanl is required for the initial assembly of the axon segment, as well as for neuronal excitability, cortical 
lamination, and neuroprotection [20]. Additionally, Mahendrasingam et al. reported that SPTAN1 is present in the cortical lattice of OHCs [11], and Legendre et al. reported that SPTAN1 is widely distributed in auditory HCs, supporting cells, and fibroblasts [18]. Of note, SPTAN1 is not only distributed along the lateral wall of HCs, but also enriched in the cuticular plate [18]. Interestingly, Liu et al. reported that spectrin forms ring-like structures around the base of stereocilia rootlets and that $\mathrm{HC}$-specific $\beta \mathrm{II}$-spectrin knockout mice display disrupted HCs polarity and profound deafness [21]. However, the role of SPTAN1 in HCs remains unknown.

In this study, we developed an HC-specific Sptanl knockout mouse model to study the function of Sptanl in HCs. The knockout mice presented with improper bundle orientation and abnormal morphology of the cuticular plate, followed by HCs loss proceeding from apex to base and more pronounced in OHCs, which resulted in early-onset deafness. In addition, Sptanl knockdown in House Ear Institute-Organ of Corti 1 (HEI-OC1) cells led to abnormal actin distribution and decreased cell spreading. Importantly, the deficiency of Sptan1 may induce the abnormal formation of focal adhesions and integrin signaling in both HEI-OC1 cells and mouse HCs. Our results suggest that Sptan1 has a major role with respect to the morphology and function of HCs through the regulation of focal adhesion signaling.

\section{Materials and Methods}

\section{Animals and Genotyping}

Sptanl knockout mice were generated at the Nanjing Biomedical Research Institute of Nanjing University (Nanjing, China), using the clustered regularly interspaced short palindromic repeat (CRISPR)-associated Cas9 nuclease (CRISPR/Cas9) genome editing technique, as previously described [22, 23]. To generate a conditional allele for Sptan 1, a targeting vector was designed to replace a $2.1 \mathrm{~kb}$ genomic fragment with loxP sites flanking exons 6,7 , and 8 of Sptan1 (Fig. S1a). The guide RNA sequence used to target the locus with Cas9 was 5S5 GGCAGCTAAGTA CGGCTCTTAGG and 3S1 AGCTAAGTCGAACAATTC AGTGG. This targeting vector construct was injected into oocytes of C57BL/6 J mice and the injected oocytes were implanted into foster female mice. The F0 generation was bred to obtain a F1 generation in which germ line transmission was confirmed. The F1 mice were genotyped using polymerase chain reaction (PCR) followed by sequence analysis. Upon crossing the mice with cochlear hair cell (HC)-specific Cre recombinase-expressing mice, exons 6-8 were excised, resulting in a short Sptanl transcript with a 218-amino acid N-terminal and one spectrin repeat. Sptan1
${ }^{f f f}$ mice were crossed with Gfil-Cre mice to produce cochlear HC-specific conditional knockout (CKO) mice (Fig. S1a). The background strains of Sptan1 mice were C57BL/6 J. Heterozygous $\mathrm{Gfi}^{\text {cre/+ }}$ mice were generated in a $129 \mathrm{~S} 6$ and $\mathrm{C} 57 \mathrm{BL} / 6 \mathrm{~J}$ mixed background as previously described, and the recombination efficiency of Gfil-Cre was approximately 93\% in HCs at postnatal day (P) 0 [24]. Male and female homozygous (Gfil-Cre ${ }^{-/+}$;Sptan $1^{\text {fff }}$ ) animals (Sptan1-CKO group) and control siblings (Gfil-Cre ${ }^{-/+} ;$Sptan $\left.1^{f /+}\right)$ (control group) were used in the present study. Mice were genotyped according to standard PCR protocols with extracted mouse tail tips [25]. The primer locations are shown in Fig. S1b. We identified Sptanl alleles using PCR primers (Table. S1), which detected Sptan1, Sptanl 3', and Sptan1 5'. The Sptan $1^{f f f}$ allele was targeted by the $f$ and $r$ primers and identified with a 300 bp band, whereas $\operatorname{Sptan}^{+/+}$was identified with a $211 \mathrm{bp}$ band. Sptan $1^{f /+}$ was identified through the simultaneous presence of $300 \mathrm{bp}$ and $211 \mathrm{bp}$ bands. Sptan $^{f /+}$ was also identified with a $1011 \mathrm{bp}$ band when using a Sptan 1 5' primer and an $1171 \mathrm{bp}$ band when using a Sptan1 3' primer (Fig. S1c). The Gfi-1F and Gfi-1R primers were used to identify the wildtype allele (609 bp), and Gfi-1F and GfilCre-R to identify the mutant allele (672 bp).

Both Sptan1-CKO and control groups contained mice with equal proportions of males and females. Animals were housed in individually ventilated cages. The temperature inside the cages was maintained at $25{ }^{\circ} \mathrm{C}$ with a relative humidity of 40-70\% and 60-65 air changes/hour in the cage. The cages including the bedding and nesting material were changed weekly. Feed and water were autoclaved prior to use.

\section{Cell Culture and Transfection}

The HEI-OC1 cell line (House Ear Institute, Los Angeles, USA), an inner ear cell line derived from the Immortomouse, expresses markers for auditory sensory cells, and is widely used to study the function of cochlear HCs. HEIOC1 cells were cultured in high-glucose Dulbecco's modified Eagle's medium (Gibco, Grand Island, MD, USA) supplemented with $10 \%$ fetal bovine serum (FBS; Gibco) and $50 \mu \mathrm{g} / \mathrm{mL}$ ampicillin (Sangon Biotech, Shanghai, China) at $33{ }^{\circ} \mathrm{C}$ under an atmosphere of $10 \% \mathrm{CO}_{2}$. Knockdown of Sptanl was performed using a lentivirus expressing either shRNA targeting Sptanl or non-relevant shRNA as a control (Hanbio Biotechnology, Shanghai, China), according to the manufacturer's instructions. The targeted sequences were as follows: Sptan 1 shRNA: 5'-CATAACTAAGGAGGCCGG CAGTGTA-3'; non-relevant shRNA: 5'-TTCTCCGAACGT GTCACGTAA- $3^{\prime}$. Briefly, $2 \mu \mathrm{L}$ shRNA was premixed with $5 \times 10^{3}$ cells during the submerged culture stage. Puromycin selection was performed to eliminate untransfected cells $24 \mathrm{~h}$ after transfection. The transfected cells simultaneously 
expressed green fluorescent protein (GFP) as a marker. We performed western blotting to verify transfection efficiency.

\section{Auditory Physiological Assessment}

Auditory brainstem responses (ABRs), distortion product otoacoustic emissions (DPOAEs), and compound action potentials (CAPs) were recorded using the Tucker-Davis hardware and BioSig software (Tucker-Davis Technology System III; TDT, Alachua, FL, USA) as previously described [26]. In total, 24 male and female mice at P30 and eight at P22 underwent auditory physiological testing. The ABR was recorded first, followed by DPOAE and CAP recordings. For all physiological tests, mice were anesthetized with intraperitoneal injections of pentobarbital sodium $(50 \mathrm{mg} /$ $\mathrm{kg}$ body weight). Mouse body temperature was maintained at $37^{\circ} \mathrm{C}$ with a thermostatic heating pad during testing and recovery from anesthesia.

For the ABR recordings, tone bursts ( $1 \mathrm{~ms}$ rise/fall with an $8 \mathrm{~ms}$ plateau from 4 to $24 \mathrm{kHz}$ at a rate of $21.1 / \mathrm{s}$ ) of stimuli were presented through a broadband speaker (FostexFT28D Dome Tweeter, Madisound, Madison, WI, USA) placed $10 \mathrm{~cm}$ in front of the mice. Evoked responses were recorded using three subdermal electrodes with the recording electrode inserted at the vertex of the skull and reference and grounding electrodes behind both pinnae. The signal was amplified $20 \times$, filtered between 300 and $3000 \mathrm{~Hz}$ with a PA16 preamplifier (TDT), and averaged over 1000 repetitions using BioSig software. The stimulus decreased from 105 to $0 \mathrm{~dB}$ sound pressure level (SPL) in 5-dB steps, and the threshold of response was judged as the lowest SPL at which a repeatable wave III was visible. If no waveform was identified at the highest presentation level (105 dB SPL) for a particular frequency, the threshold was recorded as $110 \mathrm{~dB}$ SPL.

For DPOAE measurements, two MF1 speakers (TDT) and a microphone were used to present two pure-tone stimuli and collect the distorted product, respectively, from the tested outer ear canal via a $10 \mathrm{~cm}$ length of plastic tubing. Two pure-tone stimuli at frequencies $\mathrm{f} 1$ and $\mathrm{f} 2$, with a frequency ratio (f2/f1) of 1.2 and the $\mathrm{f} 2$ level $10 \mathrm{~dB}$ lower than the $\mathrm{f} 1$ level, were generated and delivered simultaneously from 80 to $0 \mathrm{~dB}$ in $5-\mathrm{dB}$ steps. The $\mathrm{f} 2$ frequency was swept from 2.8 to $22.6 \mathrm{kHz}$ with an increase of $1 / 2$ octaves. The distorted product was amplified by $40 \mathrm{~dB}$ with an ER-10 B + low noise mic system (Etymotic Research, Inc., Elk Grove, IL, USA) before outputting to the TDT system. The DPOAE threshold was considered the lowest level of $\mathrm{f} 1$ that corresponded to a peak at 2f1-f2 that was at least $6 \mathrm{~dB}$ above the floor of the noise surrounding $2 \mathrm{f} 1-\mathrm{f} 2$.

The same stimuli were used for ABR and CAP recordings. CAPs were recorded using a silver ball electrode at the round window after surgically opening the mastoid. The grounding and reference electrodes were placed around the incision. Evoked responses were amplified using a PA16 preamplifier for CAPs (filtered below $3 \mathrm{kHz}$ ) before being averaged 100 times. The CAP amplitude was measured between the largest negative peak (N1) and the following positive peak $(\mathrm{P} 1)$. This surgical procedure received ethical approval.

\section{Immunofluorescence Staining}

Immunofluorescence (IF) staining was performed on wholemount sections, cryostat sections of the inner ears, and HEIOC1 cells. The inner ears were prepared for immunostaining using a previously described standard procedure [27]. Briefly, the inner ears were rapidly removed with the round and oval windows opened and a hole made at the apex, fixed overnight in $4 \%$ paraformaldehyde (PFA), and then decalcified in 10\% EDTA for 1 day. For whole-mount sections, the organ of Corti was dissected. For cryosections, the inner ears were dehydrated with sucrose, embedded in OCT medium, and cut into $10-\mu \mathrm{m}$ sections. HEI-OC1 cells grown on glass coverslips (WHB Scientific, Shanghai, China) were washed in PBS and fixed in 4\% PFA. The organ of Corti and cells were permeabilized with $0.5 \%$ Triton-X 100, blocked in staining blocking buffer (Beyotime Biotech, Shanghai, China), and incubated with primary antibody targeting alpha fodrin (1:200, D8B7, Abcam, Cambridge, UK), SPTBN1 (1:200, BD Biosciences, San Jose, CA, USA), myosin VIIa (1:500, Proteus BioSciences, Ramona, CA, USA), myosin 6, TRIOBP, FAK, Integrin $\beta 1$, TALIN, VINCULIN and PAXILLIN (1:200, ProteinTech Group, Chicago, USA), cleavedcaspase 3 (1:200, Cell Signaling Technology, Boston, USA) overnight at $4{ }^{\circ} \mathrm{C}$. On the second day, they were washed and incubated with Alexa Fluor ${ }^{\circledR} 488$ goat anti-mouse IgG1 ( $\gamma 1)$ (1:500 Invitrogen, Carlsbad, CA, USA), Alexa Fluor® 647 goat anti-mouse $\operatorname{IgG} 2 \mathrm{~b}(\gamma 2 \mathrm{~b})$ (1:500, Invitrogen), Alexa Fluor® 633 goat anti-mouse (1:500, Invitrogen), or Alexa Fluor® 633 goat anti-rabbit (1:500, Abcam) secondary antibodies (Table. S3) for $1 \mathrm{~h}$ at room temperature. Subsequently, they were incubated with Phalloidin-iFluor 555 reagent (1:2000; Abcam, Cambridge, UK), counterstained with Fluoroshield with DAPI histology mounting medium (Sigma-Aldrich, St. Louis, MO, USA) or DAPI $(5 \mu \mathrm{g} / \mathrm{ml}$, Beyotime Biotech) and imaged using an LSM 710 confocal microscope (Zeiss, Oberkochen, Germany) at $20 \times, 63 \times$ or $100 \times$ magnification. The images were processed and analyzed using the ZEN 2011 software (Zeiss) and the ImageJ Fiji software for calculating fluorescent intensities.

\section{Cochlear Hair Cell Count}

For quantitative evaluation of cochlear HC loss in Sptan1$\mathrm{CKO}$ and control groups, succinate dehydrogenase 
histochemistry (SDH) staining was performed as previously described [28]. Briefly, P15 and P30 mice were sacrificed under deep anesthesia; the inner ears were rapidly removed and gently perfused with freshly prepared SDH staining solution for $45 \mathrm{~min}$ at $37{ }^{\circ} \mathrm{C}$, and then fixed in $4 \%$ PFA for $24 \mathrm{~h}$ at $4{ }^{\circ} \mathrm{C}$. Whole-mount sections were prepared as described above, and the cells were examined under a light microscope (Nikon, Tokyo, Japan) at $40 \times$ magnification. The numbers of both types of HCs were counted over $0.24 \mathrm{~mm}$ intervals from the apex to the base along the entire length of the cochlea. The data were input into a custom program, and the percentages of missing IHCs and OHCs were calculated as a function of percentage distance along the entire basilar membrane based on apex to base $\mathrm{HC}$ count norms for C57 mice [29]. Data from each individual cochleogram were averaged to generate a mean cochleogram for each experimental condition.

\section{Detection of Apoptosis Using Caspase-3}

To explore the possible mechanism underlying HC apoptosis, we identified changes in the expression level of caspase-3. First, FITC-DEVD-FMK from the Caspase-3 Detection Kit (Invitrogen) was freshly prepared at a dilution of 1:100 and injected into the scala media. After $1 \mathrm{~h}$, the cochleae were then washed three times with wash buffer and fixed with ice-cold 4\% PFA in Hank's balanced salt solution overnight. Other procedures, including cochleae decalcification and cochlear membrane microdissection, were performed as described above. The specimens were then stained with DAPI to label the nuclei. Finally, the specimens were examined and imaged using a confocal microscope at $\times 63$ magnification.

\section{Western Blotting}

Ten dissected cochlear tissues and HEI-OC1 cells were washed in PBS and harvested in lysis buffer (Beyotime Biotech) supplemented with Halt Protease Inhibitor Cocktail (Thermo Fisher Scientific, Waltham, MA, USA). Whole proteins were extracted and quantified using the Bicinchoninic Acid Protein Assay Kit (Thermo Fisher Scientific). A sample of total protein $(30 \mu \mathrm{g})$ was mixed with $5 \times$ SDS loading buffer, and proteins were separated using 7.5\% SDS-PAGE (Beyotime Biotech). Proteins were transferred onto a PVDF membrane (Invitrogen) using a blotting system (Bio-Rad, Hercules, CA, USA). The membranes were blocked in 5\% non-fat dry milk for $1 \mathrm{~h}$ at room temperature and incubated with primary antibodies against alpha fodrin (1:1000; Abcam), SPTBN1 (1:1000; BD Biosciences), beta-actin or GAPDH (1:1000; Invitrogen) overnight at $4{ }^{\circ} \mathrm{C}$. On the second day, membranes were incubated with horseradish peroxidase (HRP)-conjugated secondary antibodies (1:1000, Beyotime Biotech) for $1 \mathrm{~h}$ at room temperature. The membranes were developed with enhanced electrochemiluminescence (ECL) solution (Beyotime Biotech), imaged using the ChemiDocXRS imaging system (Bio-Rad) and analyzed using the ImageJ software (NIH, Bethesda, MD, USA).

\section{Real-Time PCR}

Total RNA was extracted from four dissected cochleae using TRIzol reagent (TaKaRa Bio, Kusatsu, Japan) according to the manufacturer's instructions. The RNA concentration was determined using a NanoDrop 2000 c spectrophotometer (Thermo Fisher Scientific), and RNA integrity was evaluated using agarose gel electrophoresis and staining with ethidium bromide. RNA $(1 \mu \mathrm{g})$ was reverse transcribed to cDNA using a Quantitect Reverse Transcription Kit (Qiagen, Hilden, Germany). Real-time PCR (RT-PCR) was performed using a LightCycler® 480 II Real-time PCR Instrument (Roche, Basel, Switzerland) with a $10 \mu \mathrm{L}$ PCR mixture that included $1 \mu \mathrm{L}$ of cDNA, $5 \mu \mathrm{L}$ of $2 \times$ QuantiFast ${ }^{\circledR}$ SYBR ${ }^{\circledR}$ Green PCR Master Mix (Qiagen), $0.2 \mu \mathrm{L}$ of forward primer, 0.2 $\mu \mathrm{L}$ of reverse primer, and $3.6 \mu \mathrm{L}$ of nuclease-free water. The reactions were incubated in a 384-well optical plate (Roche) for $5 \mathrm{~min}$ at $95{ }^{\circ} \mathrm{C}$, followed by 40 cycles of $95{ }^{\circ} \mathrm{C}$ for $10 \mathrm{~s}$ and then $60^{\circ} \mathrm{C}$ for $30 \mathrm{~s}$. Each sample was run in triplicate for analysis. The primers were designed and synthesized by Generay Biotech (Shanghai, China) based on the mRNA sequence obtained from the NCBI database. All sequences of primers used for RT-PCR are shown in Table S2. GAPDH was used as an internal control. An identical threshold for fluorescence was applied for each gene of interest. Relative mRNA expression levels were calculated using the 2 $-\Delta \Delta \mathrm{Ct}$ method (threshold cycle $(\mathrm{Ct})$ ) and linearized $\mathrm{Ct}$ values before averaging [30].

\section{Scanning Electron Microscopy}

The dissected inner ears were fixed in $2.5 \%$ glutaraldehyde in phosphate buffer by intralabyrinthine perfusion overnight at $4{ }^{\circ} \mathrm{C}$. The tissues were then washed in $0.01 \mathrm{M}$ PBS, dissected to reveal the organ of Corti, and post-fixed with $1 \%$ osmium tetroxide in phosphate buffer for $2 \mathrm{~h}$ at $4{ }^{\circ} \mathrm{C}$. Postfixation, the tissues were dehydrated with a graded series of ethanol and dried using an EM CPD 300 critical point dryer with liquid $\mathrm{CO}_{2}$ (Leica, Wetzlar, Germany). The dehydrated tissues were sputter-coated with platinum $(15 \mathrm{~nm})$ using an EM SCD 050 instrument (Leica) and then analyzed using a Quanta 250 field-emission scanning electron microscope (FEI, Brno, Czech Republic). Three specimens were examined in both the Sptan1-CKO group and the control group. 


\section{Immunoprecipitation and Mass Spectrometry}

Six cochleae of each group were chopped into small pieces and lysed on ice with lysis buffer (Servicebio, China) for 30 min. Twenty microliters protein A/G agarose beads (Millipore, Temecula, CA) were added into the supernatant for $30 \mathrm{~min}$. After centrifugation, the pellet was discarded. The supernatant was immunoprecipitated using $2 \mu \mathrm{g}$ antibodies against SPTAN1 (Abcam, Cambridge, UK) or SPTBN1 (BD Biosciences, San Jose, CA) overnight at $4{ }^{\circ} \mathrm{C}$ and pulled down using fully re-suspended protein $\mathrm{A} / \mathrm{G}$ magnetic beads. The IgG was used as negative control. Samples were washed with lysis buffer and boiled, subjected to SDS-PAGE. Gels were stained with Coomassie Blue and protein bands were collected for mass spectrometry analysis. After reduction and alkylation, the samples were digested by Trypsin overnight at $37{ }^{\circ} \mathrm{C}$, and analyzed by nLC-MS/MS using a Q-Exactive mass spectrometer (Thermo Fisher Scientific) coupled with an EASY-nLC 1000 (Thermo Fisher Scientific) chromatography system, which was carried out by Shanghai Applied Protein Technology (Shanghai, China).

\section{Statistical Analysis}

Statistical analysis was performed using Sigma Plot (ver. 14.0; Systat Software Inc., San Jose, CA, USA). Normally distributed data were represented as mean \pm standard error of the mean (SEM), while non-normally distributed data were represented as medians with the $25-75 \%$ interquartile range. One-way analysis of variance (ANOVA) followed by Tukey's post hoc test for multiple comparisons were used to determine significant differences of Sptanl mRNA expression among groups. Two-way ANOVA were performed to determine significant differences in ABR, DPOAE, and CAP tests. The $\chi^{2}$ test was used to determine significant differences of the incidence of stereociliary abnormalities between Sptan1-CKO group and control group, and the percentage of rounded cell shapes between Sptan1 depletion and negative control cells. Non-parametric test was used to determine relative quantification of SPTBN1 protein by intensity analysis. $P$ values $<0.05$ were considered statistically significant. Figures were generated using the Prism software (GraphPad Software, La Jolla, CA, USA).

\section{Results}

\section{SPTAN1 Is an Abundant Component of the Cuticular Plate and Rootlets of Stereocilia}

A previous study revealed that SPTAN1 is abundant in the cuticular plate [21]. To examine the spatiotemporal expression of Sptanl in normal cochlear HCs at various stages of development, we used immunofluorescence staining (IF), real-time PCR (RT-PCR), and western blotting. SPTAN1 was abundant both in the cuticular plates and along the plasma membranes of HCs (Fig. 1a $\left(a^{\prime}-b^{\prime}\right)$ ). In addition, strong SPTAN1 expression was observed around the rootlets of stereocilia, but not in the stereocilia (Fig. 1a ( $\left.\mathrm{c}^{\prime}\right)$ ). SPTAN1 was roughly equal expressed in apical, middle, and basal turns (Fig. S2a). Moreover, SPTAN1 was co-expressed with Myosin VIIa in HCs (Fig. 1b). In addition, it was still expressed in the cortical lattices of Deiters' cells, pillar cells, and other supporting cells (Fig. S2b). Interestingly, the expression of Sptan1 at both the mRNA and protein levels was decreased during development (Fig. 1c-d). In summary, our results show that HCs are enriched in SPTAN1, specifically localized in the cuticular plate and rootlets of stereocilia.

\section{Sptan 1-CKO Mice Develop Hearing Loss}

We examined the resulting auditory physiological properties, including the auditory brainstem responses (ABRs), distortion product otoacoustic emissions (DPOAEs), and compound action potentials (CAPs) in two groups. The ABR thresholds for clicks and 4, 8, 16, and $24 \mathrm{kHz}$ at P22 were $76.25 \pm 8.20,90 \pm 0,75 \pm 3.54,70 \pm 6.12,87.5 \pm 2.5 \mathrm{~dB}$ SPL in Sptan 1-CKO mice $(n=4)$, respectively, compared to $23.75 \pm 2.17,51.25 \pm 11.39,18.75 \pm 7.40,13.75 \pm 4.15$, $35 \pm 3.54 \mathrm{~dB}$ SPL in control mice $(n=4)$, respectively. The results showed significantly elevated thresholds at clicks and 4, 8, 16, and $24 \mathrm{kHz}(P<0.001)$ in Sptan1-CKO mice (Fig. 2a). The ABR thresholds for clicks and 4, 8, 16 , and $24 \mathrm{kHz}$ at $\mathrm{P} 30$ were $75.80 \pm 8.86,103.33 \pm 3.73$, $82.50 \pm 2.50,77.50 \pm 7.50$, and $90.00 \pm 11.55 \mathrm{~dB}$ SPL in Sptan1-CKO mice $(n=6)$, respectively, compared to $29.17 \pm 1.86,40.00 \pm 4.08,23.33 \pm 4.71,36.67 \pm 14.04$, and $76.66 \pm 6.87 \mathrm{~dB}$ SPL in control mice $(n=6)$, respectively. The results showed significantly elevated thresholds at clicks and $4,8,16(P<0.001)$, and $24 \mathrm{kHz}(P<0.01)$ in Sptan 1 CKO mice (Fig. 2b). The ABR raw traces of the two groups at $\mathrm{P} 30$ are shown in Fig. 2c.

At P30, the DPOAE thresholds of Sptan1-CKO mice were also significantly increased compared to those of control mice at 4, 5.6, 8, $11.3(P<0.001)$, and $16 \mathrm{kHz}(P<0.01)$ (Fig. 2d). Similarly, the CAP thresholds were significantly increased $(P<0.001)$ (Fig. 2e), and the CAP amplitudes were significantly decreased from 70 to $105 \mathrm{~dB}$ SPL at $8 \mathrm{kHz}$ in Sptan1-CKO versus control mice $(P<0.05)$ (Fig. 2f).

\section{HCs of Sptan 1-CKO Mice Show Stereocilia and Cuticular Plate Defects}

Next, we performed phalloidin staining, IF, and scanning electron microscopy (SEM) to investigate changes in the 
a
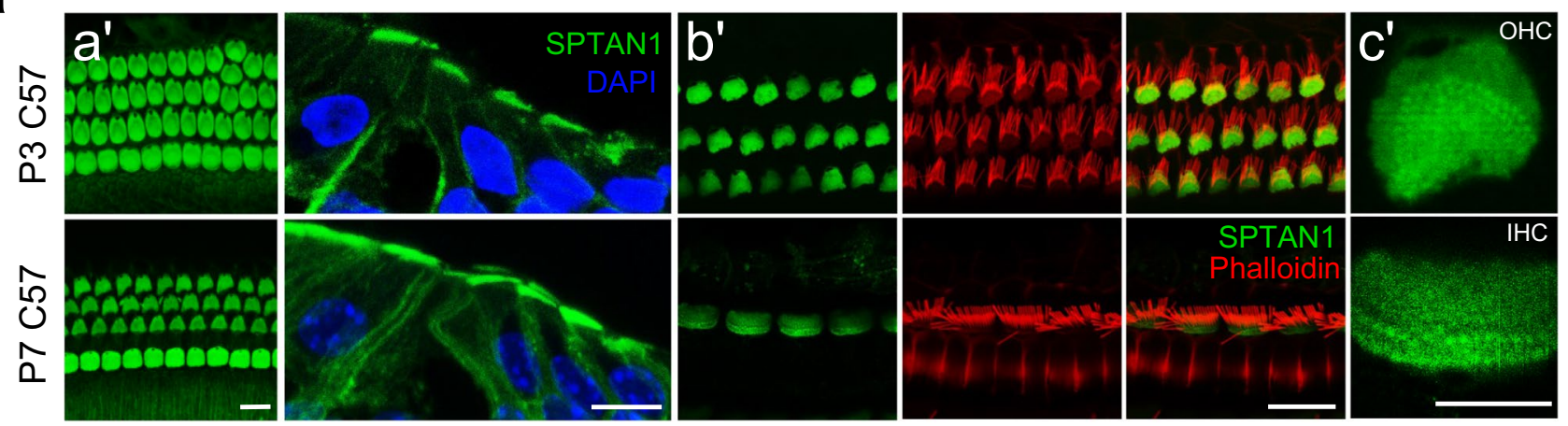

b
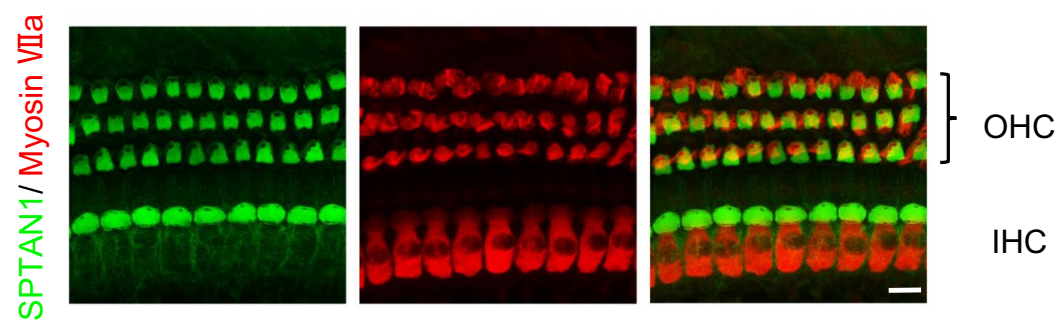

C

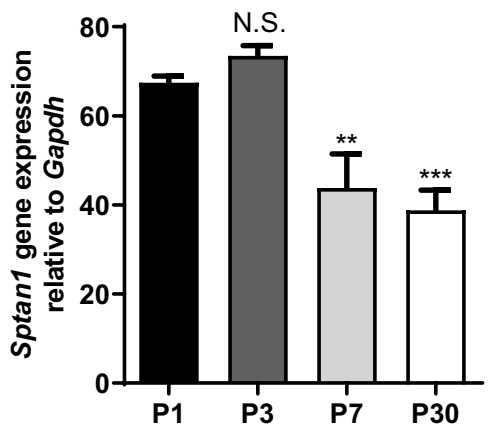

d

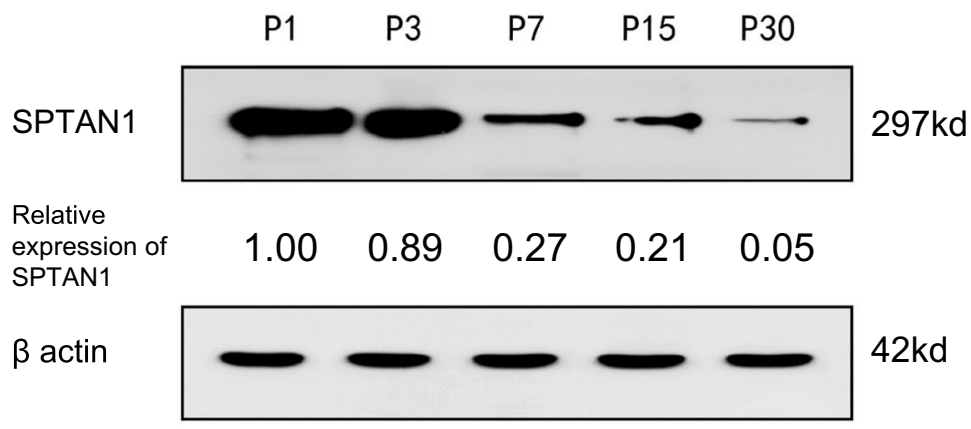

Fig. 1 Sptanl is an abundant component of the cuticular plate and rootlets of stereocilia. a (a') Immunofluorescence of SPTAN1 in whole-mount staining and cryostat sections of the cochleae of P3 and P7 C57 BL/6 J mice. Scale bar: $10 \mu \mathrm{m}$. (b') Immunofluorescence of SPTAN1 in whole-mount staining in guinea pigs. SPTAN1 (green) is mainly expressed in the cuticular plate and along the plasma membrane of hair cells. Scale bar: $10 \mu \mathrm{m}$. (c') SPTAN1 is expressed in rootlets of stereocilia of OHC and IHC. Scale bar: $2.5 \mu \mathrm{m}$. b SPTAN1 co-expresses with Myosin VIIa in hair cells in P15 mice. Scale bar:
$10 \mu \mathrm{m}$. c RT-PCR analysis of Sptan1 mRNA expression in the organ of Corti of C57BL/6 J mice ( $n=6)$. The expression of Sptan1 at P3 is similar to P1, and the expression at P7 and P30 is significantly lower than P1. One-way analysis of variance (ANOVA) followed by Tukey's post hoc test for multiple comparisons were used to determine significant differences among groups. Error bars represent standard error of the mean. $* * P<0.01, * * * P<0.001$. d Analysis of SPTAN1 expression by western blotting showing higher levels at P1 and P3 than at P7, P15, and P30 in the organ of Corti

In addition, flattened and misshapen bundles and loss of stereocilia were also defined as abnormal stereocilia [31]. OHCs displayed a V-shape hair bundle in control mice. However, the stereocilia of OHCs from Sptan 1-CKO mice lost their polarity and displayed unusual shapes, such as " $\mathrm{C}$ " and "S" shapes, or showed a disrupted staircase architecture, such as the fusion of stereocilia (Fig. 3a-c). Of note, abnormalities in the stereocilia rotation of $0^{\circ}$. Others were defined as mis-oriented bundles. 
Fig. 2 Sptan1-CKO mice at P22 and $\mathrm{P} 30$ show elevated hearing thresholds. a Sptan1-CKO mice show increased ABR thresholds ( $n=4$, red columns) compared to those of control mice $(n=4$, black columns) at clicks and 4 , 8, 16, $24 \mathrm{kHz}$ at P22. b Sptan1CKO mice show increased ABR thresholds ( $n=6$, red columns) compared to those of control mice $(n=6$, black columns) at clicks and 4, 8, 16, $24 \mathrm{kHz}$ at P30. c Representative ABR traces recorded from $\mathrm{P} 30$ control $(n=3)$ and Sptan 1-CKO mice $(n=3)$, with an $8-\mathrm{kHz}$ tone burst between 55- and 90-dB SPL. d DPOAE thresholds for pure-tone stimuli in Sptan1CKO ( $n=6$, red line) are significantly higher than those in control animals except at 2.8 and $22.6 \mathrm{kHz}$ at $\mathrm{P} 30(n=6$, black line). e Sptan1-CKO mice show higher CAP thresholds $(n=6$, red columns) than control mice ( $n=6$, black columns) at P30. e Sptan1-CKO mice ( $n=6$, red line) show lower CAP amplitudes from 70 to $105 \mathrm{~dB}$ SPL at $8 \mathrm{kHz}$ than control mice $(n=6$, black line) at P30. The two-way ANOVA was used to determine significant differences in $\mathbf{a}, \mathbf{b}, \mathbf{d}, \mathbf{e}$, and $\mathbf{f}$. Error bars represent standard error of the mean. $* P<0.05, * * P<0.01$, $* * * P<0.001$ a

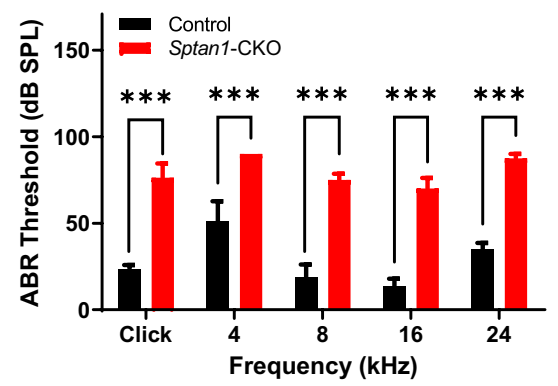

C

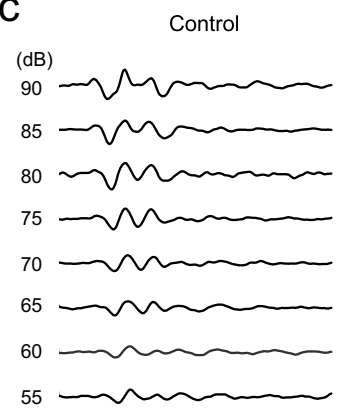

b

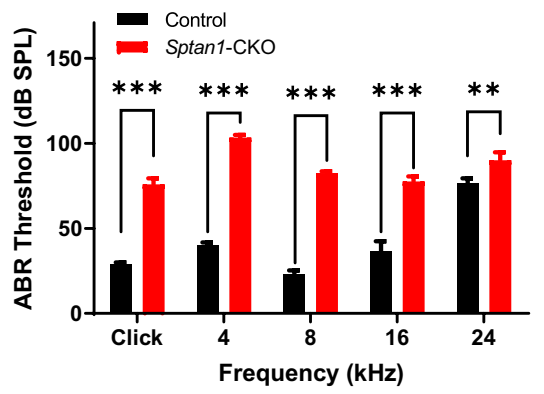

d

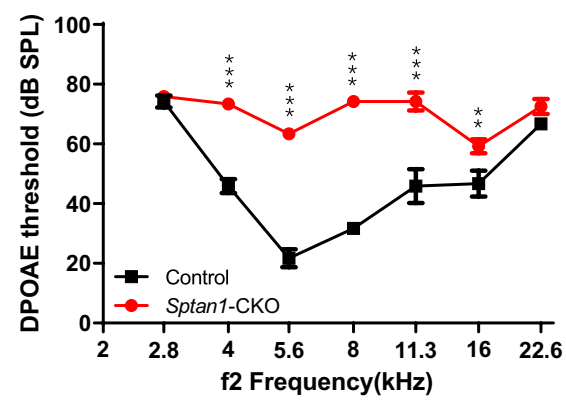

f

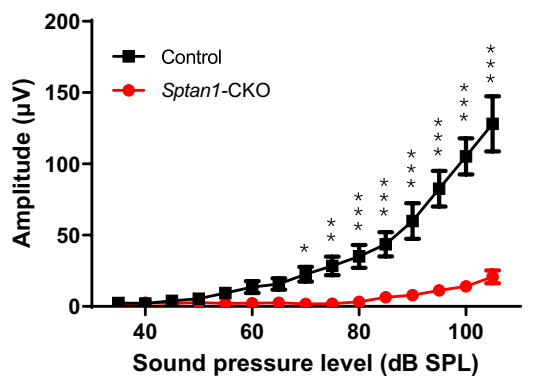

of OHCs in Sptan1-CKO mice increased with age, with loss of stereocilia observed at P15 and P30 (Fig. 3d), and more severe dysmorphologies in apical and middle regions than basal region (Fig. 3a). Additionally, the shapes of the cuticular plates (indicated by blue circles) were also abnormal in some OHCs from $\mathrm{P} 3$ (round or oval shapes), and more OHCs presented with abnormal cuticular plates at $\mathrm{P} 30$ (Fig. 3c). The incidence of stereociliary abnormalities of $\mathrm{OHCs}$ throughout entire length of the cochlear basilar membrane at different ages is significantly higher $(P<0.001)$ in Sptan 1-CKO versus control mice (Fig. 3d). Previous study reported that TRIOBP was necessary to form mature stereocilia rootlets; it was essential for the biogenesis of rootlets that provided durable flexibility at the taper and mechanical rigidity to the stereocilia bundle. Then, to confirm the abnormal rootlets of stereocilia in Sptan 1CKO mice, we further performed IF of TRIOBP at P3, P7, $\mathrm{P} 15$, and P30. Mice in the control group had normal V-shaped rootlets in OHCs whereas in Sptan1-CKO mice, rootlets were misoriented from P3 (Fig. 4a-d), and some even lost from P15 (Fig. 4c-d). In line with these results, while the rootlets of IHCs stereocilia were not lost until P30, there were slightly disordered (Fig. 4d).

We further investigated the changes in $\mathrm{HC}$ morphology using myosin VIIa, a specific marker of HCs. The morphology of most OHCs became irregular, such as round or polygons shapes in Sptan1-CKO mice at P15 (Fig. S3a), an alteration that was further aggravated at P30. In addition, a massive loss of OHCs occurred at P30; the surviving cells were those in which the Cre recombinase was too weak to knockout SPTAN1 (Fig. S3b).

\section{The Abundance of Hair Cells Is Decreased in Sptan 1-CKO Mice Due to Apoptosis}

To determine whether Sptanl deficiency affected $\mathrm{HC}$ abundance, HCs were counted using Myosin 6 in 
a

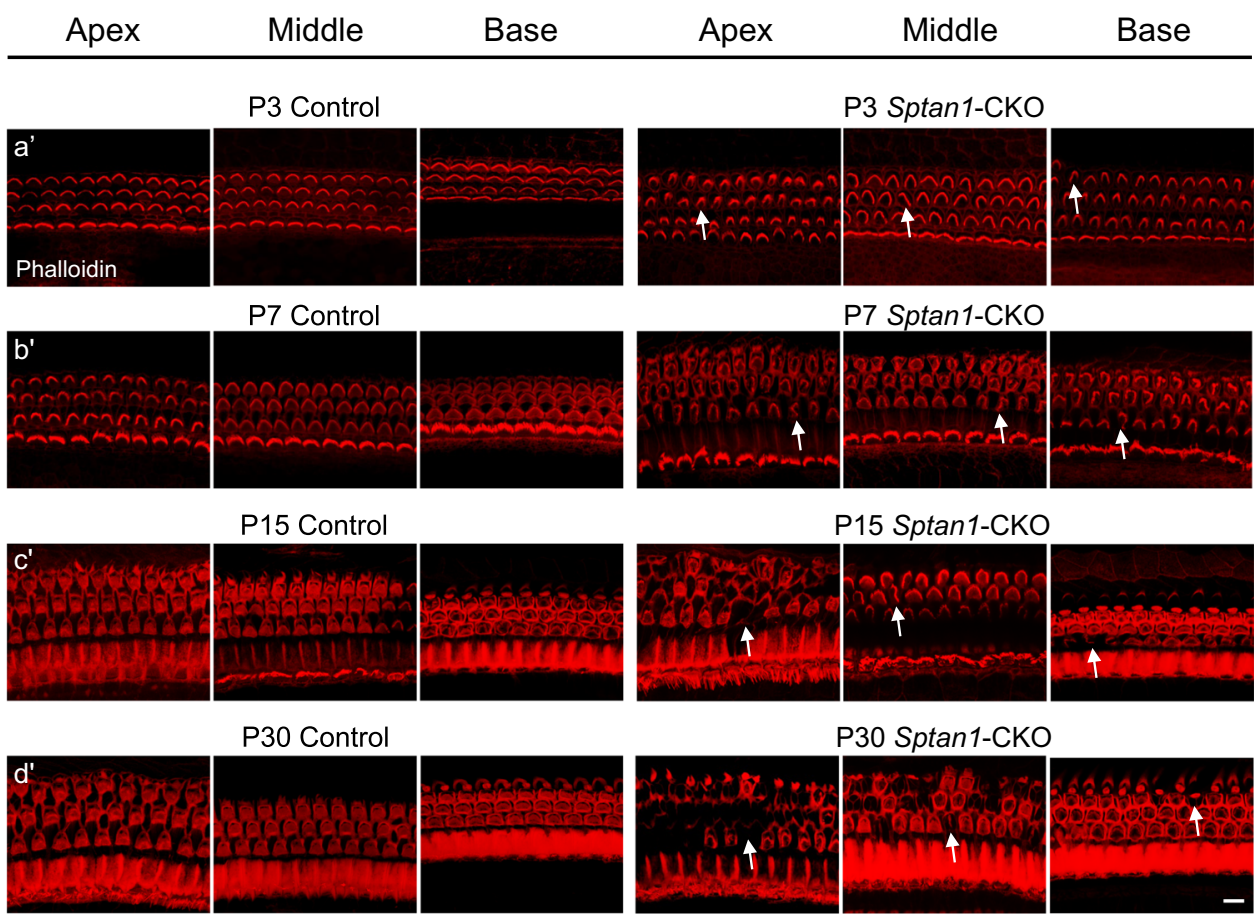

Fig. 3 Outer hair cells (OHCs) of Sptan1-CKO mice exhibit severely altered stereocilia and cuticular plate morphologies. a (a') At P3, in control mice, the OHCs show normal V-shaped hair bundles, whereas in Sptan1-CKO mice, some stereocilia exhibit "U" and "C" shapes or partially missing. (b') At P7, there are more abnormal stereocilia than at P3 in Sptan1-CKO mice. (c') At P15, some stereocilia show abnormal shapes in Sptan1-CKO mice and some even lost. (d') At P30, the loss of hair bundles was more severe than at P15 in Sptan1CKO mice. The loss of stereocilia was more severe in apical and middle regions than basal region. Abnormal stereocilia bundles are highlighted by arrows. Scale bar: $10 \mu \mathrm{m}$. b Higher magnification of stereocilia in OHCs. Scale bar: $5 \mu \mathrm{m}$. c (a-f) Low-magnification SEM images of cochlear hair bundles from mice of different genotypes and ages. The stereocilia of OHCs started to lose their polarity as early as at P3. The extent of the disruption is larger at P7. Some loss is apparent at P30. The abnormal shapes of stereociliary bundles are high-

Sptan1-CKO and control mice at P3, P7, P15, and P30. No $\mathrm{HCs}$ were lost at $\mathrm{P} 3$ and $\mathrm{P} 7$, while $\mathrm{OHCs}$ were apparently lost at P15 and P30 (Fig. S4). Next, we used succinate dehydrogenase histochemistry (SDH) to quantitatively evaluate the HC loss in both groups at $\mathrm{P} 15$ and $\mathrm{P} 30$. The mean cochleograms ( $n=6$ in each group at each stage) indicated that $\mathrm{OHCs}$ were rapidly and progressively lost in Sptan1-CKO mice. At P15, up to $40 \%$ of OHCs were lost at the apical-middle turns, whereas a few were lost in the middle-basal turns. At P30, more severe OHC loss occurred, with approximately $80 \%$ of OHCs loss at the apical-middle turns and 5\% loss at the middle-basal turns (Fig. 5a-c). Of note, the IHC abundance was unaffected at P15 and P30 (Fig. 5a-c, S2-3). Similar results were lighted by yellow arrows, and stereocilia loss is indicated by asterisks. In Fig. 3cf, the stereocilium indicated by the left yellow arrow is significantly fused, without staircase architecture, compared to that of other stereocilia. Scale bar: $20 \mu \mathrm{m}$. $\left(\mathrm{a}^{\prime}-\mathrm{f}^{\prime}\right)$ High-magnification SEM images of OHCs hair bundles from mice of different genotypes and ages. The shapes of cuticular plates became oval in Sptan1-CKO mice, as highlighted by the blue circles. $\left(\mathrm{a}^{\prime \prime}-\mathrm{f}^{\prime \prime}\right)$ High-magnification SEM images of inner hair cells (IHCs) hair bundles from mice of different genotypes and ages. Images of different turns of the cochleae were captured. $\mathbf{a}, \mathbf{b}, \mathbf{c}$, and $\mathbf{d}$ represent apical-middle turns, and $\mathbf{e}$ and f represent middle-basal turns. Scale bar: $5 \mu \mathrm{m}$. d The incidence of stereociliary abnormalities of OHCs throughout entire length of the cochlear basilar membrane at different ages is significantly higher in Sptan1-CKO versus control mice, as per the $\chi^{2}$ test. At each time point, $\sim 600 \mathrm{OHCs}$ from 3 mice in each group were analyzed. Error bars represent standard error of the mean. $* * * P<0.001$

observed in the P60 mouse cochlea, with the majority of OHCs absent in the apical-middle turns (Fig. S5).

We then investigated whether OHCs underwent programmed cell death. HCs were labeled with DAPI for the visualization of the nuclei at $\mathrm{P} 30$. The HCs of control mice exhibited characteristic cell nuclei shapes, i.e., large and round nuclei (Fig. 5d), whereas the nuclei of Sptan1CKO mice were darkly stained and severely shrunken, or fragmented (Fig. 5d). Furthermore, cochleae were labeled with cell-permeable fluorogenic probes specific to caspase- 3 to determine if the programmed cell death was caspase-mediated. Caspase- 3 was not activated in control mice, whereas positive caspase-3 labeling was observed in Sptan1-CKO mice (Fig. 5d). 
b

P3 Control P3 Sptan1-CKO

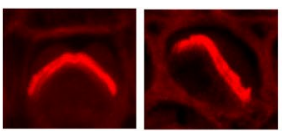

P15 Control P15 Sptan1-CKO P30 Control P30 Sptan1-CKO
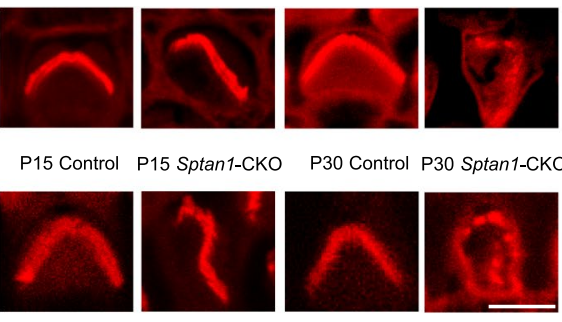

d

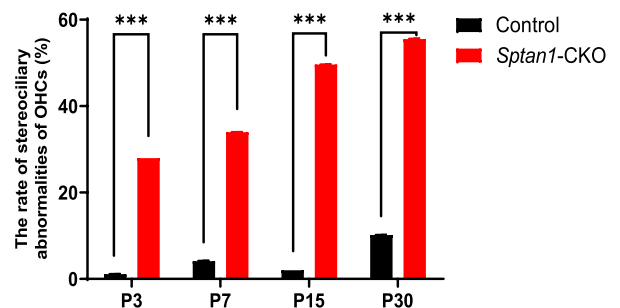

C

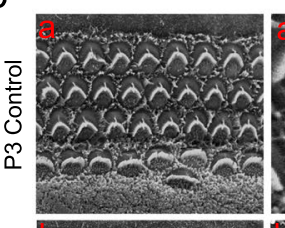

$\mathrm{OHC}$

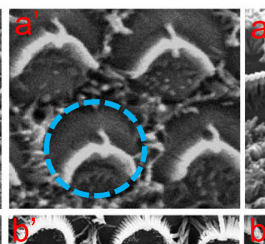

$\mathrm{IHC}$
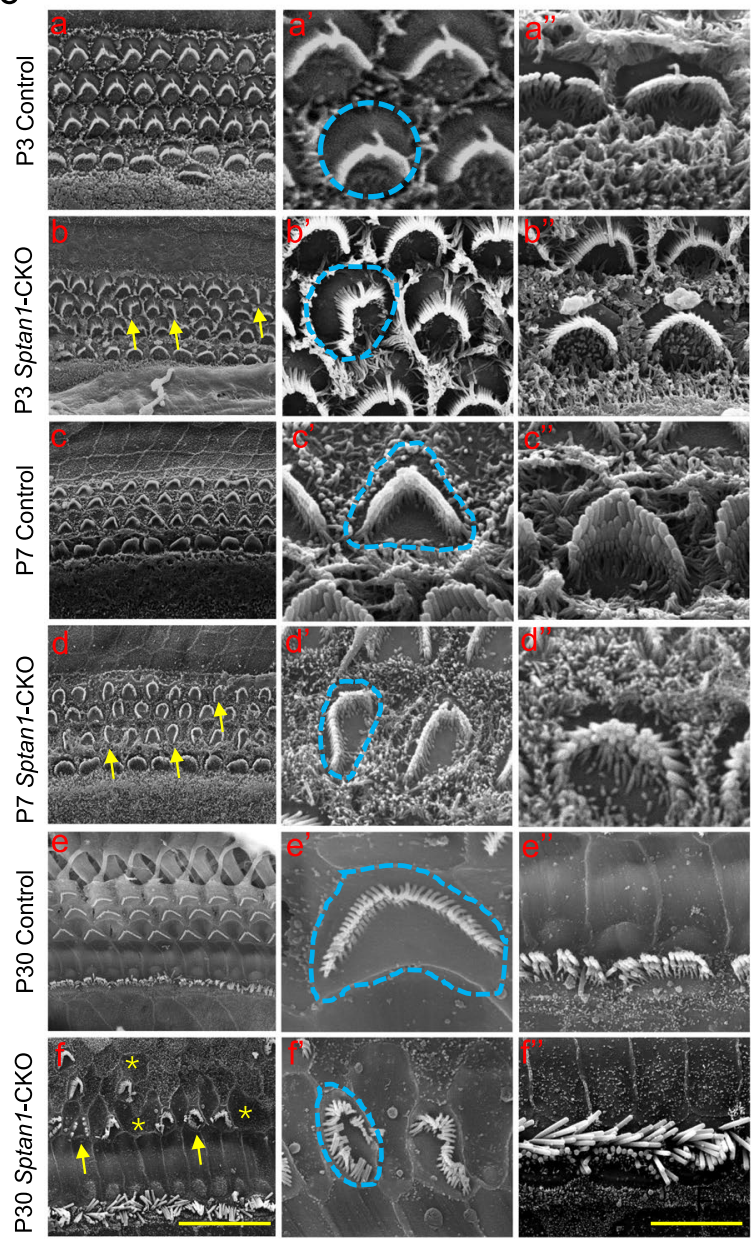

Fig. 3 (continued)

\section{The Cuticular Plates of Sptan 1-CKO Mice Show Decreased/Absent SPTBN1 Expression}

Previous studies have reported that SPTAN1 and SPTBN1 interact with each other in the cuticular plates of cochlear HCs in mice [21]. Therefore, we examined the expression of SPTBN1 in Sptan1 knockdown HEI-OC1 cells and Sptan 1-CKO mice. The results indicated that Sptan1 knockdown led to decreased levels of SPTBN1 in HEI-OC1 cells (Fig. 6a). Furthermore, the expression of SPTBN1 also differed in Sptan1 depletion (sh-Sptan1) group from small hairpin negative control (sh-NC) cells (Fig. 6b). Then, SPTAN1/SPTBN1 immunofluorescence staining was performed with whole-mount basilar membranes at P15. SPTAN1 and SPTBN1 were co-expressed in the cuticular plate with the same distribution in control mice but absent or downregulated (both proteins) in Sptan1-CKO mice (Fig. 6c). 
Fig. 4 The rootlets of stereocilia are abnormal in Sptan1-CKO mice of different ages. The organ of Corti was stained with anti-TRIOBP (green) antibodies and Phalloidin (red). In the control group, the rootlets of stereocilia of OHCs are V-shaped. a At P3, in Sptan1-CKO mice, some rootlets of stereocilia of OHCs are deformed. b At P7, there are more misorientated rootlets of stereocilia of $\mathrm{OHCs}$ than at P3 in Sptan1-CKO mice. c At P15, abnormal rootlets of stereocilia of OHCs are increased in Sptan1-CKO mice; some degree of loss is also presented. d At P30, more rootlets of stereocilia loss is evident in Sptan1-CKO mice (versus P15). The rootlets of stereocilia of IHCs are also slightly disordered at P30. All images were captured in the middle turns. Scale bar: $10 \mu \mathrm{m}$ a

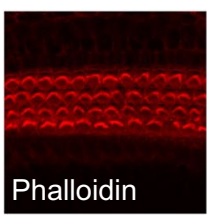

P3 Control

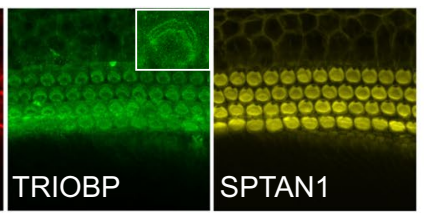

b

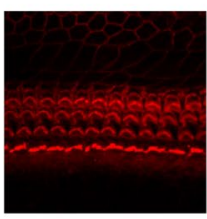

P7 Control
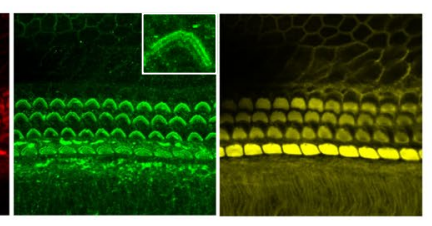

P15 Control
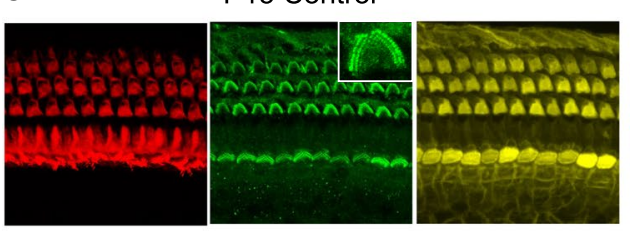

d

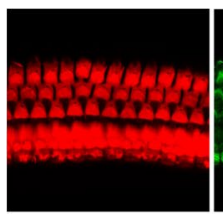

P30 Control
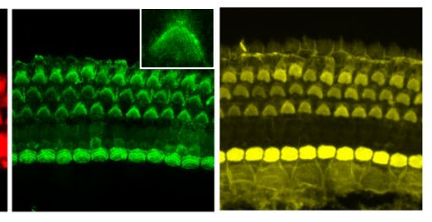

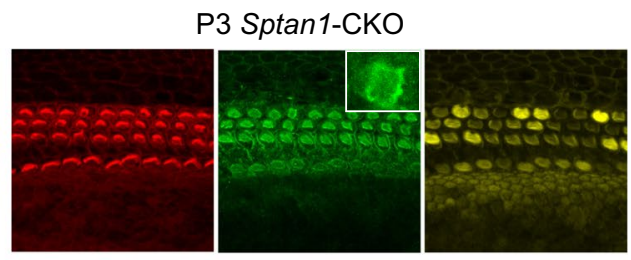

P7 Sptan1-CKO

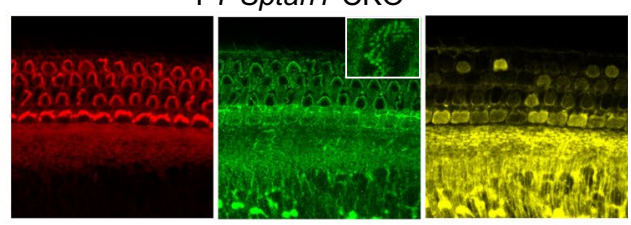

P15 Sptan1-CKO

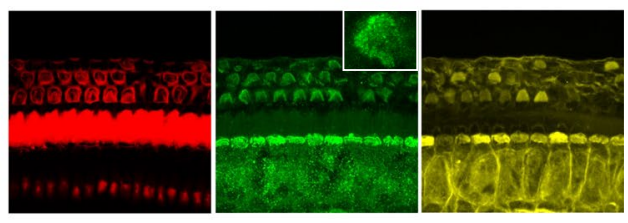

P30 Sptan1-CKO
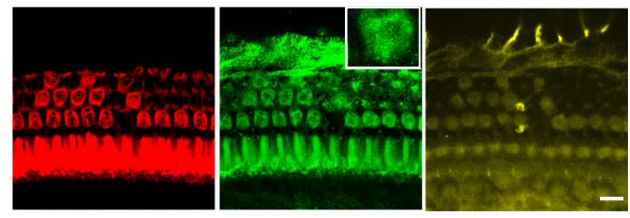

\section{Sptan 1 Plays a Role Through Regulating Focal Adhesion Signaling}

The actin cytoskeleton is critical for the maintenance of cell shape and abundance in the cuticular plate. The altered morphology of the cuticular plate in Sptan1-CKO mice prompted us to further examine the changes in cell shape and spreading. F-actin of sh-NC cells was mainly located in the cytoplasm, and stress fiber-like filaments were clearly observed (Fig. 7a). Sh-NC cells also manifested various shapes, including fusiform or polygonal cell shapes, and extended flat membrane protrusions (lamellipodia), and fingerlike protrusions (filopodia) (Fig. 7a-b). However, depletion of Sptan1 caused cell rounding; a large portion of the cells retracted their protrusions (Fig. 7a-b, and f). Furthermore, marked changes in the actin cytoarchitecture were observed, including the loss of distinct arc-shaped actin bundles (Fig. 7a). Of note, we performed IF of cleavedcaspase 3 to confirm that the abnormal shape of sh-Sptan 1 cells was not due to the loss of viability (Fig. 7c) and quantitatively analyzed the percentage of rounded cell shapes in two groups. In line with the above results, this quantitative analysis revealed that the number of rounded cells in the sh-Sptan1 group was significantly higher $(P<0.001)$ than that in the sh-NC group (Fig. 7d). In addition, we analyzed the proteins that may interact with SPTAN1 and SPTBN1 using mass spectrometry analysis. We found that SPTAN1 co-precipitated with SPTBN1, and SPTBN1 coprecipitated with Integrin $\beta 1$, TALIN-1, and VINCULIN (Table. S4). Cell adhesion and spreading require integrinmediated formation of focal adhesions, and the structural modifications of focal adhesions require the assistance of focal adhesion kinase (FAK), PAXILLIN, VINCULIN, and TALIN to mediate the interaction between the extracellular matrix (ECM) and the actin cytoskeleton (Fig. 7e). Integrins promote the formation of signaling complexes that regulate F-actin accumulation, which is best characterized by focal adhesions containing FAK, and it has been reported that FAK was located at the apical HC surface and in stereocilia. Therefore, we further examined whether the depletion of Sptanl caused its inability to bind to focal adhesion-related proteins, which led to abnormal actin cytoskeletal structure and decreased cell spreading in the sh-Sptanl group. Here, we studied expression of proteins including FAK, Integrin $\beta 1$, PAXILLIN, TALIN, and VINCULIN in sh-NC and shSptan 1 groups. Integrin $\beta 1$ and FAK were co-localized with SPTAN1 (Fig. 7f $\left(a^{\prime}-b^{\prime}\right)$ ). In sh-NC group, these proteins were expressed in nucleus and cytoplasm, whereas in the shSptan1 group, morphology of these focal adhesions-related proteins was disrupted, which led to destruction of formation 
Fig. 5 Loss of hair cells in Sptan1-CKO mice at different ages. a At P15, Sptan1-CKO mice show a loss of up to $40 \%$ of outer hair cells (OHCs) at the apical-middle turns, but not at the middle-basal turns. At $\mathrm{P} 30$, more severe $\mathrm{OHC}$ loss is observed in Sptan1-CKO mice, with no inner hair cells (IHCs) loss. b Succinate dehydrogenase histochemistry (SDH) staining of hair cells in Sptan1-CKO mice at P15. c SDH staining of hair cells showing OHCs loss in Sptan1-CKO mice at P30. Scale bar: $25 \mu \mathrm{m}$. d At P30, no caspase-3-positive OHCs and IHCs are observed in control mice. The large and round nuclei (blue) of normal hair cells in control mice are indicated by circles. However, OHCs from Sptan1-CKO mice show the presence of caspase- 3 activation (green) and shrunken and fragmented nuclei, as highlighted by the arrows. The middle turns of the cochlea were used in $\mathbf{d}$. Scale bar: $10 \mu \mathrm{m}$ a

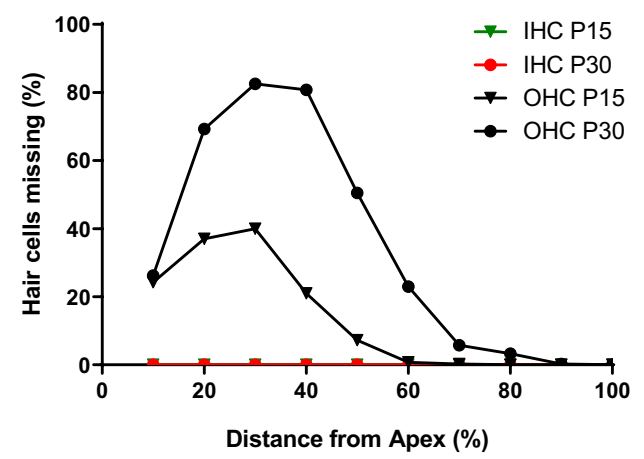

b
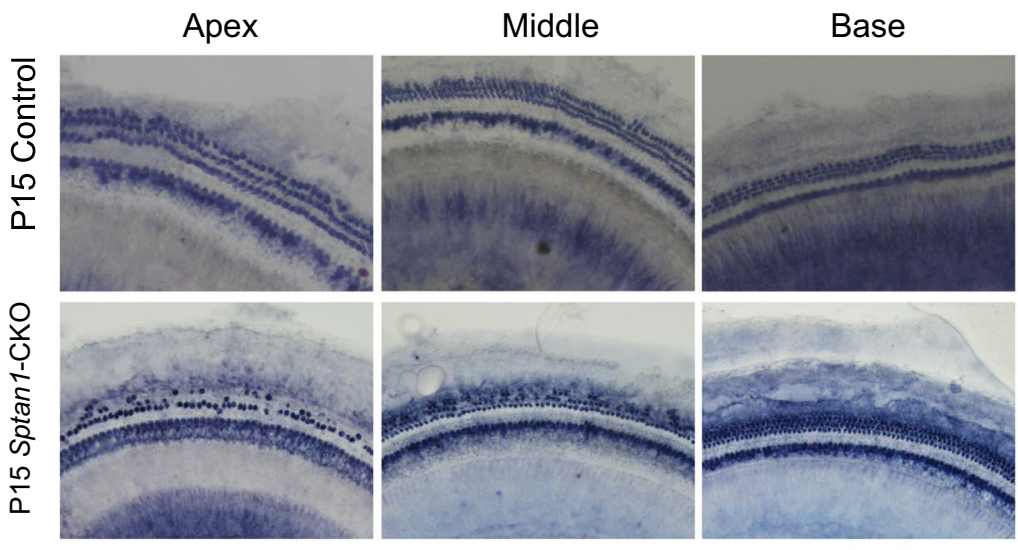

C
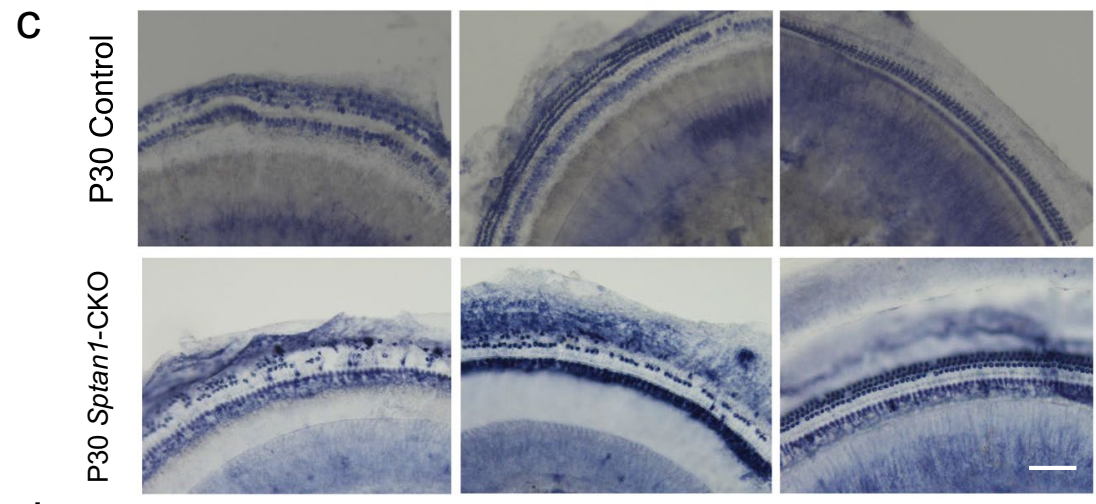

O
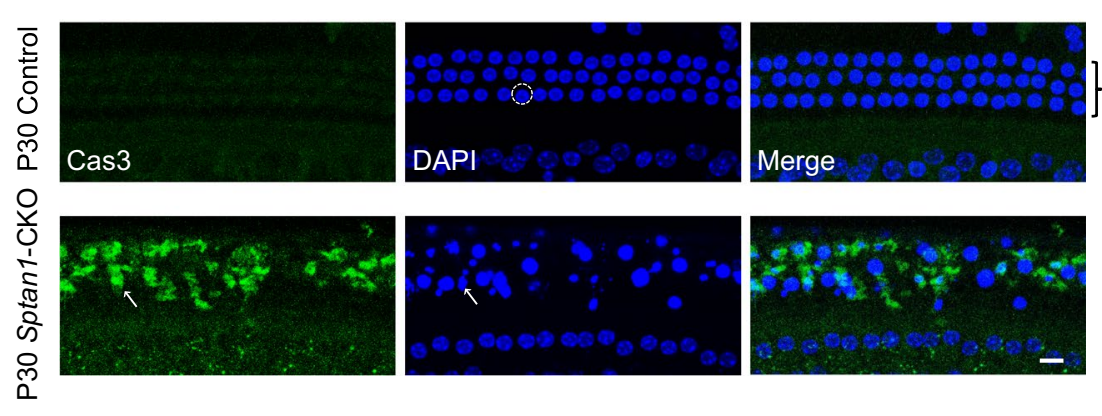

$\mathrm{OHC}$

IHC
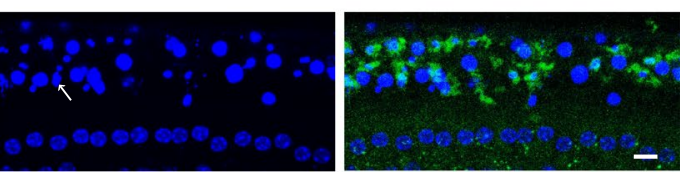

and maintenance of cytoskeletal architecture including stress fibers (Fig. $\left.7 f\left(c^{\prime}\right)\right)$.

Furthermore, for the sake of validation, we examined the formation of integrin-focal adhesions in P7 and P15 mice. In Sptan1-CKO mice, Integrin $\beta 1$ and FAK were absent in the cuticular plate at P7 (Fig. 8a, d) and P15 (Fig. 8b-c, e-f). The absence of Integrin $\beta 1$ and FAK in the cuticular plates occurred at P7, and the HCs were not missing at this time (nucleus still existed) (Fig. 8a, d). Therefore, we have reached the conclusion that reduced Integrin $\beta 1$ and FAK in the cuticular plates were related to the knockout of Sptan1, but not because of missing of HCs. Additionally, the abnormal 
Fig. 6 SPTBN1 is decreased or absent in Sptanl knockdown HEI-OC1 cells and in HCs from Sptan1-CKO mice. a Representative western blotting showing the protein levels of SPTAN1 and SPTBN1. The SPTAN1 and SPTBN1 levels are decreased in Sptan1-depleted (sh-Sptan1) versus control (sh-NC) cells. GAPDH was used as the loading control. b $\left(\mathbf{a}^{\prime}-\mathbf{b}^{\prime}\right)$ Cells in the sh-Sptan1 show decreased SPTAN1 and SPTBN1 levels compared to those in sh-NCtreated cells. Scale bar: $10 \mu \mathrm{m}$. $\left(c^{\prime}\right)$ Relative quantification of SPTBN1 protein by intensity analysis. Data are shown by means \pm SEM. $* P<0.05$. c In Sptan1-CKO mice, the absence of SPTAN1 is associated with the absence of SPTBN1 in cochlear hair cells at P15. Scale bar: $10 \mu \mathrm{m}$ a

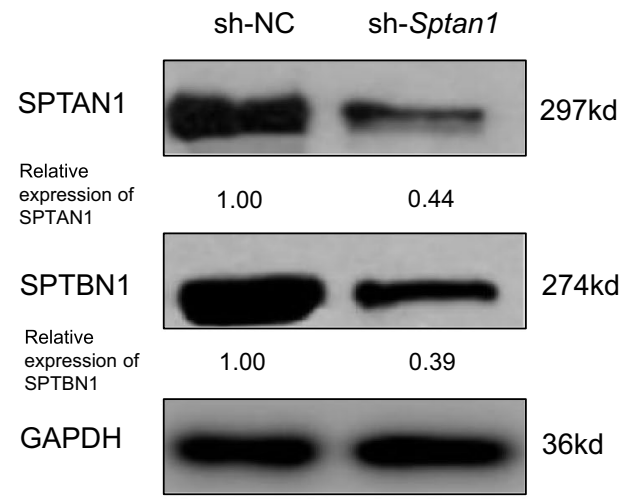

b
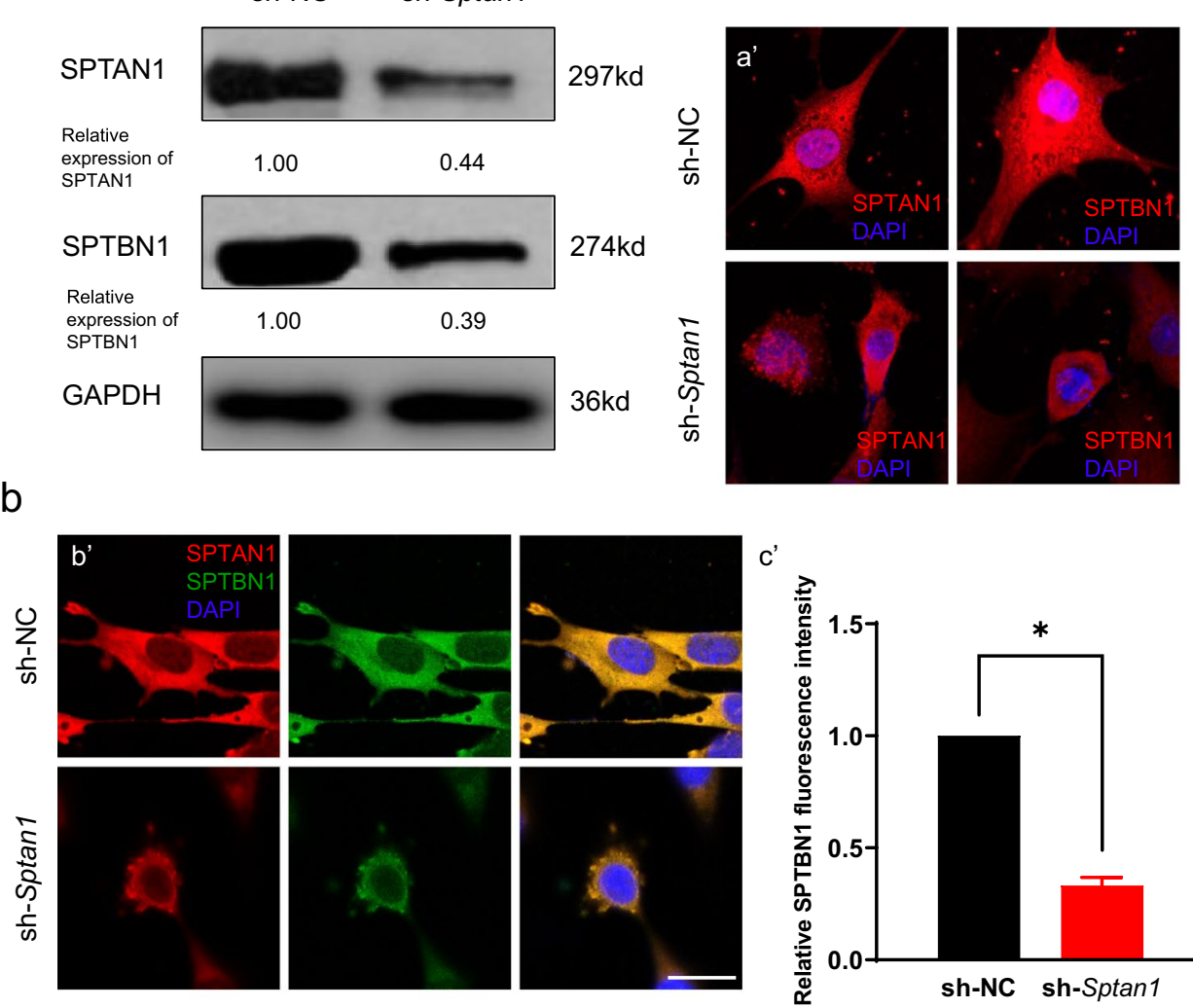

b

\section{.}
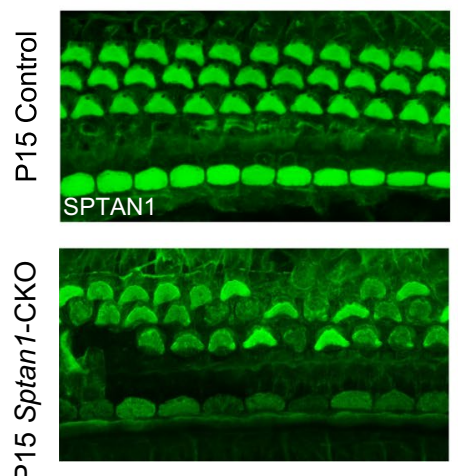
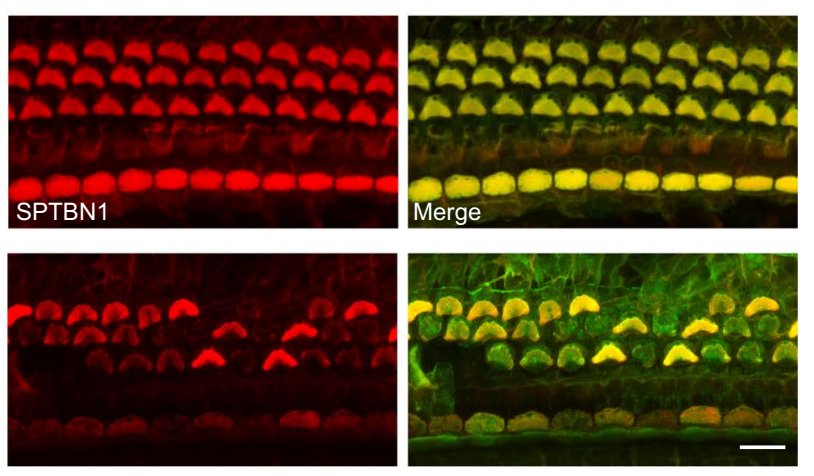

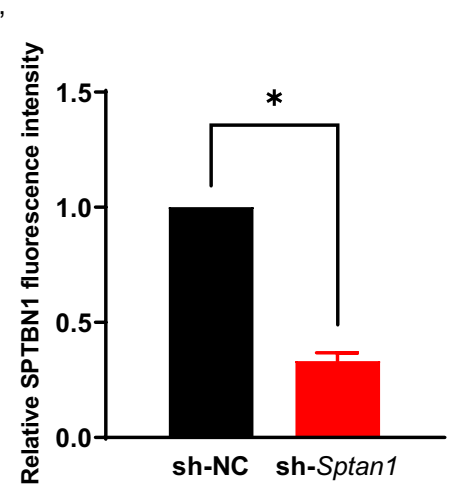

aggregation or absence of PAXILLIN (Fig. 8g) and VINCULIN (Fig. 8h) at P15 were also observed. Therefore, we concluded that the knockout of Sptanl led to the absence of integrin-focal adhesion signaling, and it might indirectly bind to focal adhesion-related proteins by binding to SPTBN1 to play the role in hair cell morphology and function.

\section{Discussion}

Together, using a HC-specific Sptanl knockout mouse model, we found that Sptanl is essential for maintaining stereocilia and cuticular plate morphologies. Sptan l-CKO mice presented with rapid OHC loss and early-onset deafness. Additionally, shRNA-mediated Sptanl knockdown in HEIOC1 cells led to abnormal actin distribution and decreased cell spreading. Importantly, the deficiency of Sptanl induced the abnormal formation of focal adhesions and integrin signaling in both HEI-OC1 cells and mouse HCs. In summary, the results of these in vivo and in vitro studies suggest that Sptanl is indispensable for the maintenance of normal cuticular plate and stereocilia and the consequent survival of cochlear HCs via focal adhesion signaling.

In HCs, SPTAN1 was expressed in cuticular plate, rootlets of stereocilia, and along the lateral wall of HCs. In addition, it was still expressed in the cortical lattices 
Fig. 7 Sptan1 knockdown HEIOC1 cells exhibit altered cell shapes and decreased spreading. a F-actin organization in HEIOC1 cells. Phalloidin labeling is intense in the cytoplasm, and stress fiber-like filaments are clearly observed in control (shNC) cells. In Sptan1-depleted (sh-Sptan1) cells, phalloidin labeling is present in both the cytoplasm and cortex of the cell body, with the loss of stress fiber-like filaments. Scale bar: $10 \mu \mathrm{m}$. b Compared to sh-NC cells, sh-Sptan 1 cells show decreased spreading. Scale bar: $50 \mu \mathrm{m}$. c The cell viability of sh-Sptan1 and sh-NC cells is comparable. Scale bar: $50 \mu \mathrm{m}$. d The percentage of rounded cell shapes in the sh-Sptan1 group is significantly higher than that in the sh-NC group $(\sim 350$ cells in each group). Error bars represent standard error of the mean. $* * * P<0.001$ e The illustration of proteins involved in focal adhesions. f $\left(\mathrm{a}^{\prime}\right)$ The co-localization of SPTAN1 and Integrin $\beta 1$ in sh-NC and sh-Sptanl groups. ( $b^{\prime}$ ) The co-localization of SPTAN1 and FAK in sh-NC and sh-Sptanl groups. (c') Control cells extend flat membrane protrusions (lamellipodia) and fingerlike protrusions (filopodia) whereas focal adhesions are disrupted in sh-Sptan1 cells. The components of focal adhesion are gathered around the nucleus. Scale bar: $10 \mu \mathrm{m}$ a
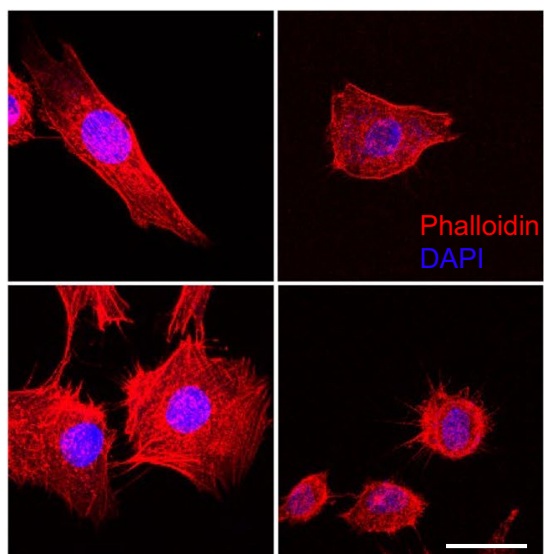

sh-NC

C

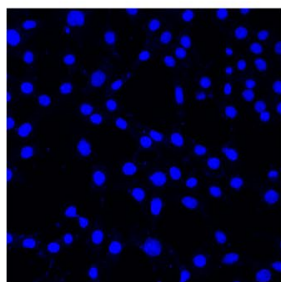

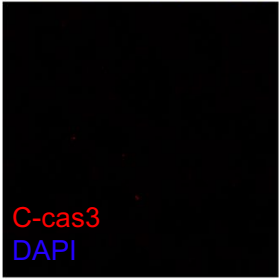

sh-NC

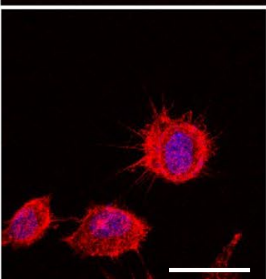

sh-Sptan1
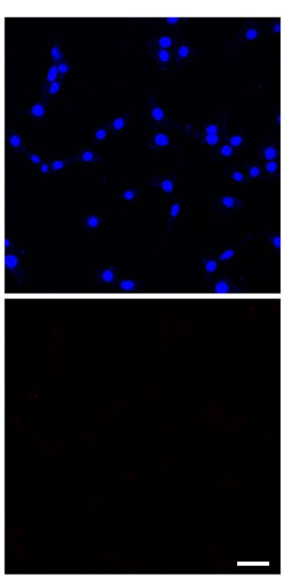

sh-Sptan1 b
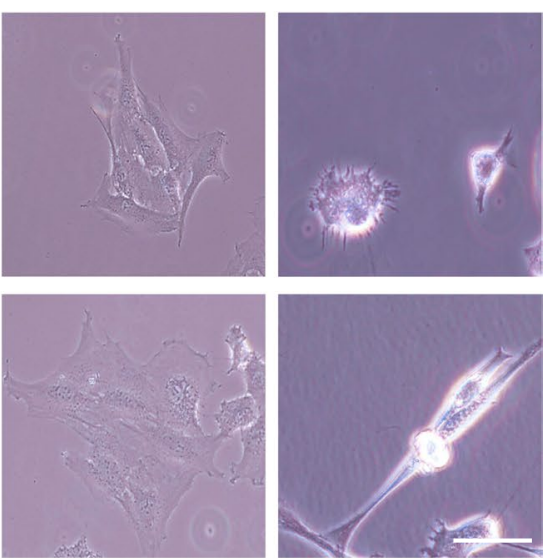

sh-NC
sh-Sptan1

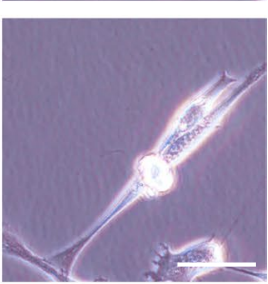

d

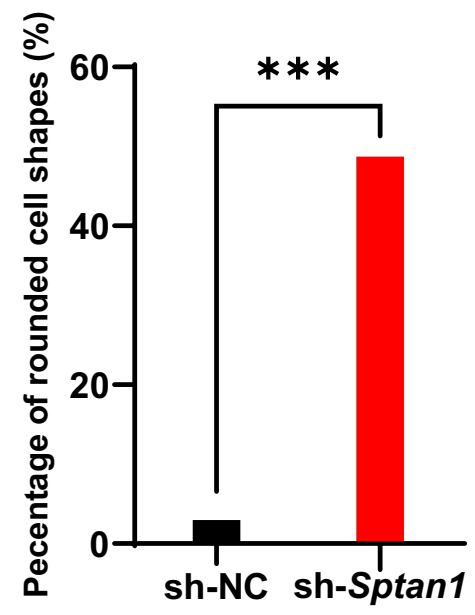

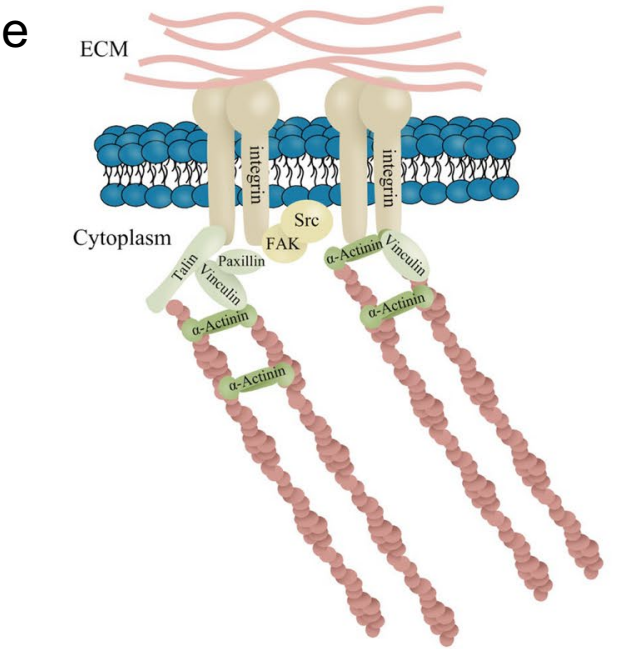

of Deiters' cells, pillar cells, and other supporting cells. The expression changes detected by RT-PCR and western blotting could also reflect supporting cells changes in part. Previous study reported that in the cochlea of mouse, rootlets began to develop around E15, and the cuticular plate then forms around the rootlet by E18 [32]. Another study observed rootlets development in mice from P1, with the upper rootlet appearing first and then extending down 
Fig. 7 (continued)

f
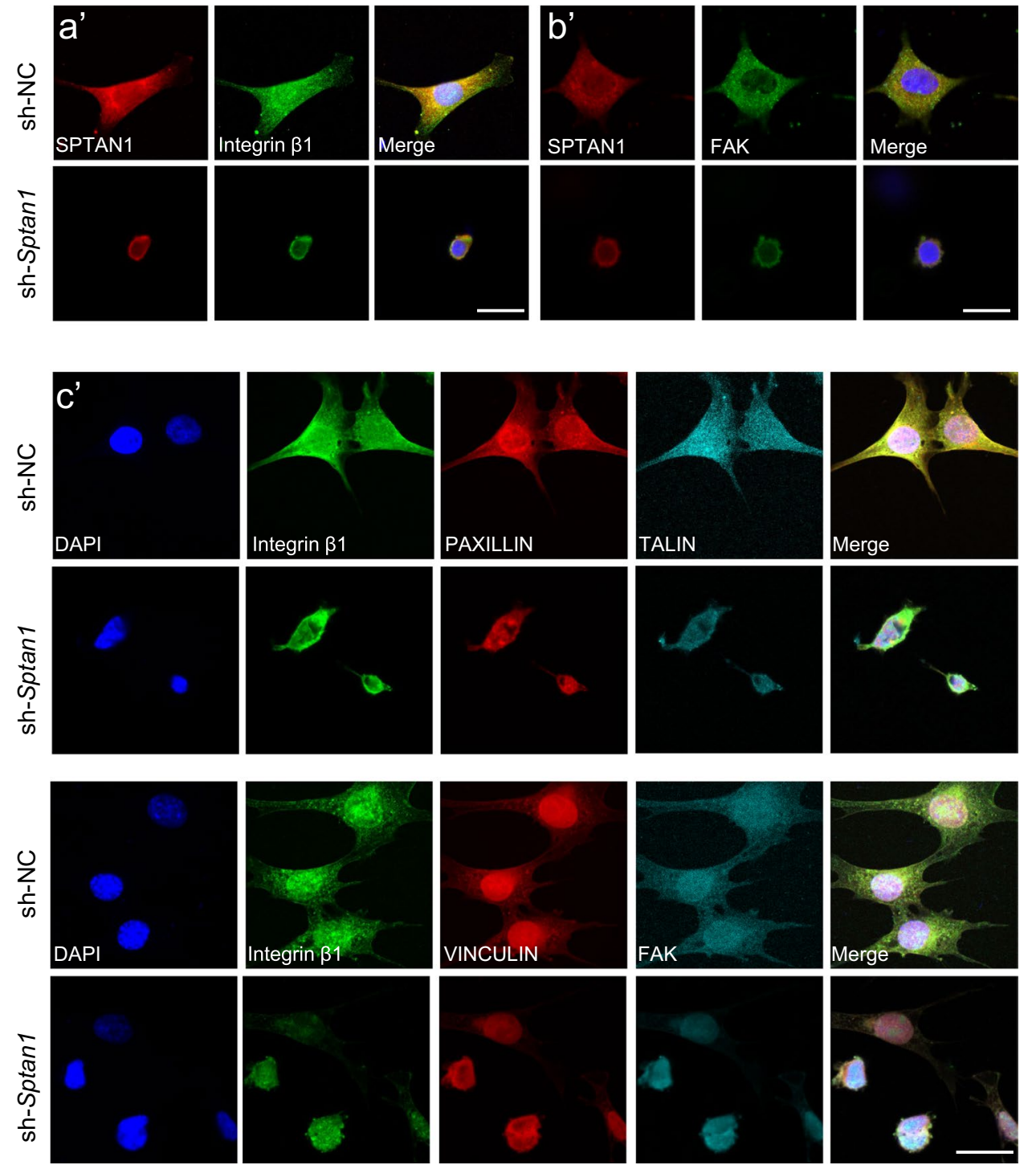

into the cuticular plate [33]. The SPTAN1 was abundantly expressed in the early postnatal stages, which supported the idea that SPTAN1 in the HC is essential for rootlets and cuticular plates development. In addition, although it started to decrease at around P7, it was still abundantly expressed at P15 and P30, which suggested that it may play an essential role in maintaining the morphology of stereocilia and cuticular plate during late postnatal stages. As global Sptan1 knockout mice are embryonic lethal [34], we used the GfilCre mouse line as a tool to generate HC-specific Sptan1 deficient mice, to define the roles of Sptanl in cochlear HCs. We found some degree of mosaicism in the context of the Gfil-Cre ${ }^{-/+}$-mediated recombination in HCs in the context of different ages, likely due to the low expression level of $\mathrm{Cr}$ in those cells. Of note $\mathrm{G} f \mathrm{I} 1-\mathrm{Cr} \mathrm{C}^{-/+} ; \mathrm{Sptan}^{\mathrm{flf}}$ mice presented with decreased expression levels of Sptan1 in most
IHCs and OHCs at birth as well as a progressive loss of this protein with age. Previously, the recombination efficiency of Gfil-Cre in cochlear hair cells was shown to be approximately $93 \%$ [24], and our study showed comparable rates of approximately $81 \%$. Importantly, we cannot exclude the possibility that the timing of Gfil-Cre expression is slightly different in different types of hair cells, which may account for the differential effective rates [35]. In spite of the different efficiency in OHCs with a decreased or absent expression of Sptan 1, the stereocilia and cuticular plates lost their normal appearance. Although that study reported that heterozygous GfI ${ }^{\mathrm{Cre}}$ mice also exhibited early-onset progressive hearing loss, auditory thresholds were not significantly affected, at least in the range of 8 to $16 \mathrm{kHz}$ for up to 3 months after birth. In contrast, in our study, Sptanl-CKO mice showed a low-frequency hearing loss, suggesting that this animal 
Fig. 8 The loss of Sptan1 affects focal adhesion signaling in P7 and P15 mice. a Cryosections showing the absence of Integrin $\beta 1$ from the cuticular plate in P7 Sptan1-CKO mice. b Cryosections showing the absence of Integrin $\beta 1$ from the cuticular plate in P15 Sptan1CKO mice. c Whole-mount staining showing a decreased fluorescence intensity of Integrin $\beta 1$ in P15 Sptan1-CKO mice. d Cryosections showing the absence of FAK from the cuticular plate in P7 Sptan1CKO mice. e Cryosections showing the absence of FAK from the cuticular plate in $\mathrm{P} 15$ Sptan1-CKO mice. f Wholemount staining showing the abnormal aggregation and distribution of FAK in P15 Sptan1CKO mice. $g$ Whole-mount staining showing the abnormal aggregation and distribution of PAXILLIN in P15 Sptan1-CKO mice. $\mathbf{h}$ Whole-mount staining showing the abnormal aggregation and distribution of VINCULIN in P15 Sptan1-CKO mice. The middle turns of the cochlea were used. $\mathrm{c}$ and $\mathrm{f}-\mathrm{h}$ represent the expression of proteins in different focal planes including stereocilia bundle, cuticular plate, and nucleus of the hair cells. Scale bar: $10 \mu \mathrm{m}$
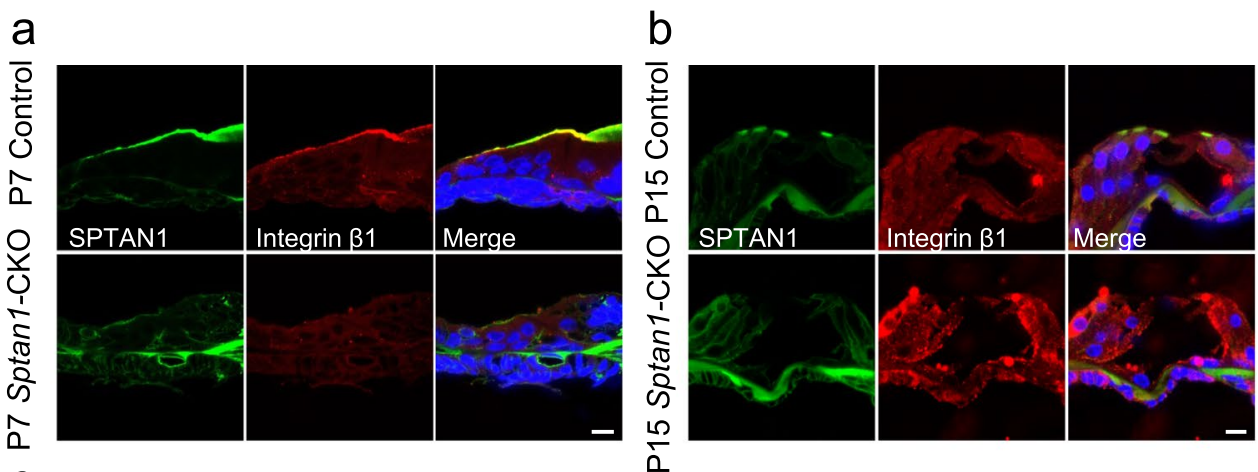

C
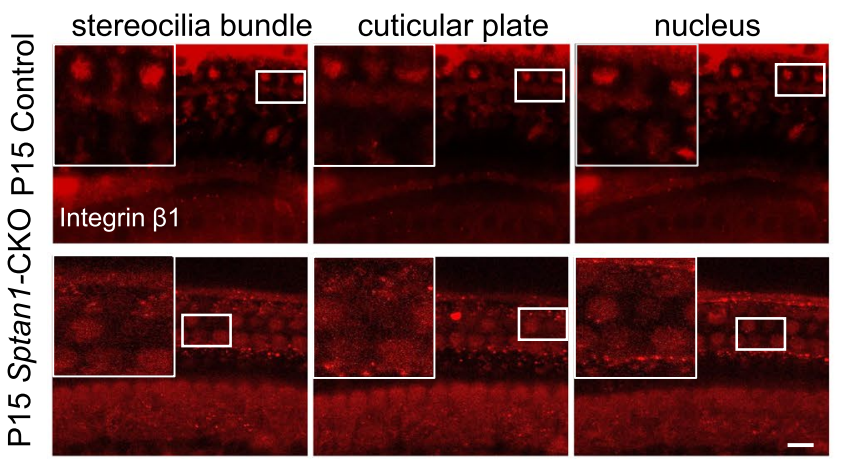

d

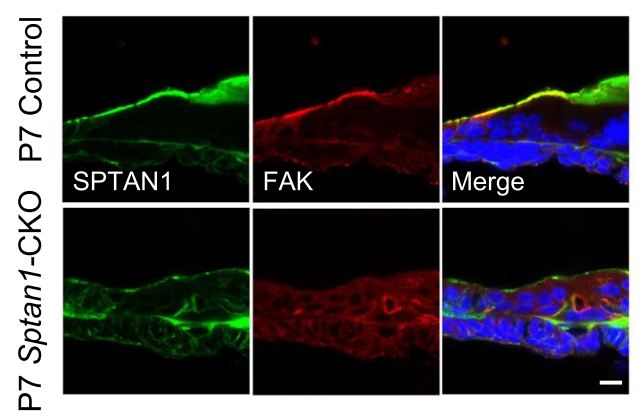

e
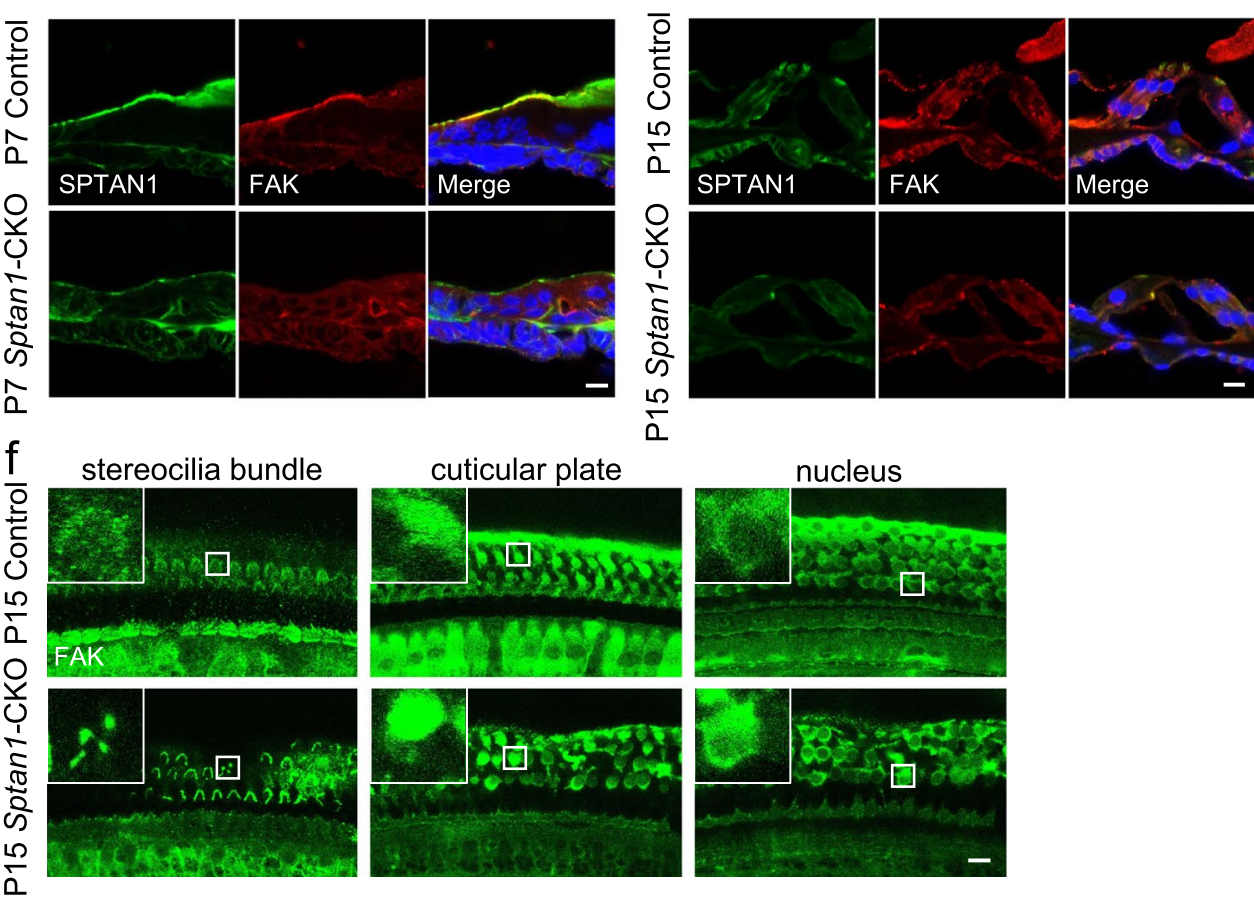

cuticular plate

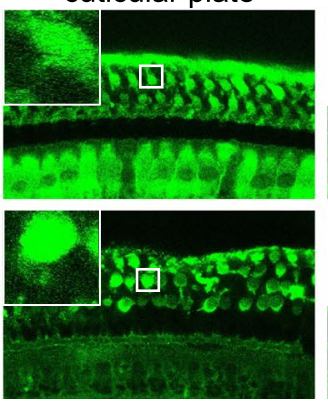

nucleus

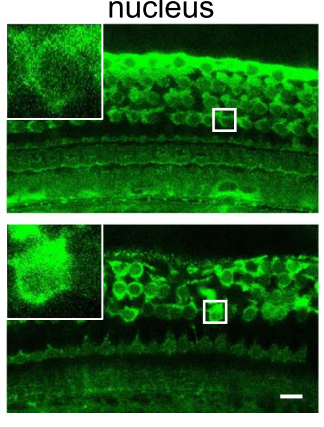

model is suitable for studying the correlation between Sptan1 expression and changes in cochlear HC morphology and auditory function. In our study, hearing loss was assessed in far-field recording using ABRs, and in acute, near-field recordings of the CAP. DPOAE was used to assess the function of OHCs. The thresholds of ABR and CAP were slightly different (maybe due to operations differences); however, the results all showed that hearing thresholds at different frequencies were elevated in Sptan1-CKO group compared to the control group. They all proved that the Sptan1-CKO mice developed hearing loss, and these were in consistent with the morphological evidence.

Sptanl deficiency in OHCs of Sptan1-CKO mice was associated with abnormal stereocilia and cuticular plate 
Fig. 8 (continued)

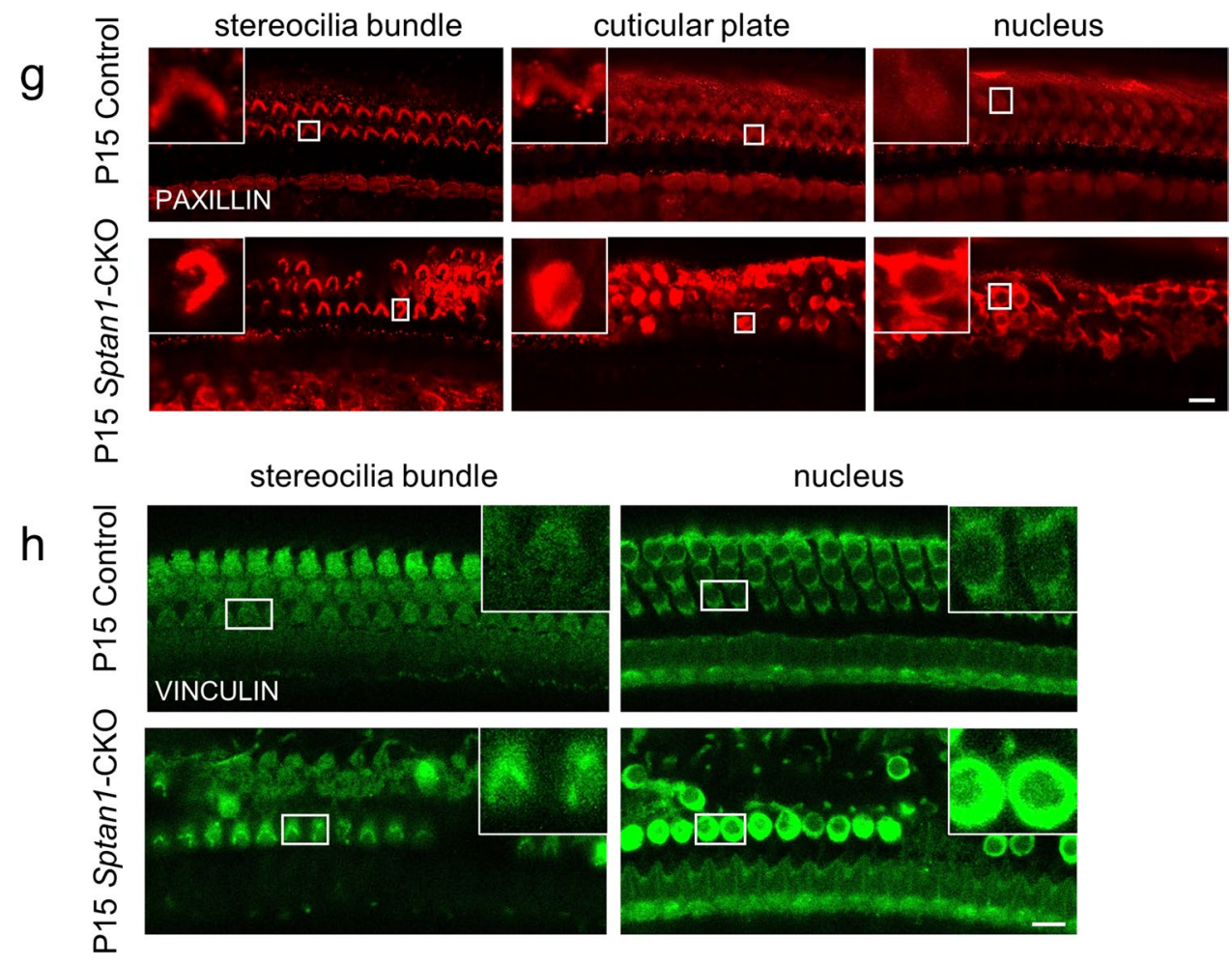

morphology. The cuticular plate is enriched with F-actin [7]. Sptan 1 deficiency led to flattened morphological changes in the cuticular plates, which may affect the distribution and state of F-actin. Therefore, we performed in vitro studies via the shRNA-mediated Sptanl knockdown in HEI-OC1 cells. Interestingly, Sptan1 knockdown HEI-OC1 cells showed shrunken sizes, disorganized F-actin, and loss of stress fiber-like filaments, indicating that Sptan1 is crucial for the maintenance of the shape of cultured HEI-OC1 cells through collaboration with F-actin. In addition, the deletion of Sptan1 led to the decreased expression of SPTBN1. Previous study reported that spectrins function as tetramers that consist of two $\alpha$ and two $\beta$ subunits [36], and the spectrin tetramers line the internal side of the plasma membrane, where they interact with integral membrane proteins and F-actin [37]. In cochlear HCs, SPTAN1 and SPTBN1 form a cylindrical structure in the rootlet region of each stereocilium, probably important for the shearing of mechanical stress during deflection [21]. In addition, the results of mass spectrometry analysis showed that SPTAN1 co-precipitated with SPTBN1, which suggested that SPTAN1 and SPTBN1 co-localized in the rootlets of stereocilia and cuticular plates and bound to each other to stabilize the stereocilia. HC-specific Sptbnl knockout mice displayed severe hearing loss and exhibited more severe phenotypes in the context of $\mathrm{OHC}$ stereocilia compared to that of IHC stereocilia [21], which was consistent with the phenotypes of HC-specific Sptan 1 knockout mice. Moreover, Liu and colleagues reported that SPTAN1 was still found specifically in the cortical lattice region of HCs in Sptbnl knockout mice [21], whereas SPTBN1 was absent in Sptan1-deficient cochlear HCs and HEI-OC1 cells in our study. Therefore, altogether, our results suggest that SPTAN1 may be required for the specific localization and function of SPTBN1 in the cuticular plate of HCs; SPTAN1 is likely an essential component of the direct or indirect maintenance of stereocilia. In addition, compared with other regions, the low-frequency region was affected more severely in Sptan1-CKO mice. This may be due to the higher efficiency of Gfil-Cre recombinase-mediated recombination in the apical turns, more HCs lacked the expression of Sptan1, which led to more HCs loss. The difference further proved that Sptanl was essential for cochlea hair cell morphology and function. However, the specific mechanism behind this particular phenotype needs to be further studied.

In addition to anchoring stereocilia directly at the rootlet, our findings suggest that Sptan1 plays a role in the maintenance of the morphology and function of stereocilia and $\mathrm{HCs}$ via the regulation of integrin-focal adhesion signaling. Integrin-tethered focal adhesions participate in mechanosensing, structure, and signaling [38]. Integrins can interact with the ECM to provide cells with support and survival signals [39]. Mutations in ECM components, such as type IV collagen, can lead to hereditary hearing loss, and integrins may be involved in this process. Additionally, sensory HCs have an elaborate F-actin cytoskeleton, and integrins can regulate the dynamics of actin. In fact, a previous study reported that 
$\alpha 8 \beta 1$ integrin are proteins that are required for normal hearing and stereocilia structure, stereocilia did not achieve complete maturation or were not maintained after the knockout of integrin $\alpha 8$ [40]. Another study demonstrated that integrin $\alpha 8$ and protocadherin- 15 act as a complex to regulate cilia biogenesis in sensory cells [41]. Therefore, we speculate that Sptan1 might interact with Integrin $\beta 1$ to maintain the stereocilia in the cuticular plate. Of note, integrins promote the formation of signaling complexes that regulate $\mathrm{F}$-actin accumulation, which is best characterized by focal adhesions containing FAK [42]. FAK is a key component of integrin activated signaling pathways that is recruited to the apical hair cell surface during stereocilia formation [40, 43]. Previous study reported that in the noise exposed organ of Corti, FAK p-Tyr577 was detected in OHC stereocilia of noise damaged regions. Excessive noise-induced mechanical stress of stereocilia induced FAK phosphorylation in stereocilia [44]. These data indicate that $\alpha 8 \beta 1$ integrin and FAK are in a molecular pathway that regulates the assembly or maintenance of the cytoskeleton of the stereocilia. Another study reported that TALIN was expressed in anchoring cells that could create and/or maintain tension on the spiral ligamentbasilar membrane complex and influence the mechanical properties of the basilar membrane [45]. Importantly, our results suggested that SPTAN1 indirectly interacted with focal adhesion-related proteins, and the knockout of Sptan 1 caused the abnormal aggregation or absence of FAK, PAXILLIN, TALIN, and VINCULIN. Therefore, we conclude that Sptan 1 may affect actin dynamics through focal adhesion signaling, resulting in stereocilia formation and maturation disorders. Of note, focal adhesions had not actually been observed in our study so the link remains speculative, and more experimental verification should be carried out to explore the mechanisms that govern both the direct and indirect interactions between Sptanl and the components of the signaling complexes.

The rapid death of HCs is secondary to Sptanl deficiency. Defects in any of the sensory cells of the organ of Corti including IHCs, OHCs, the tectorial membrane, supporting cells, or cells of the stria vascularis can lead to deafness. In our study, loss of OHCs is the major cochlear pathologies in early-onset hearing loss. Sptan1-CKO mice demonstrated rapidly progressive $\mathrm{OHC}$ loss starting from the apex, with the most severe $\mathrm{OHC}$ loss at the apical-middle turns observed as early as P15; additionally, IHC loss was observed until P60. Damage patterns progressing from partial to complete OHCs lesioning and eventually to the IHCs. The different fates of both types of HCs were reported in previous studies $[33,46]$. The OHCs are one of the most vulnerable components in most pathologies [47, 48]. In our study, though the expression of SPTAN1 in IHCs and OHCs appeared similar, the loss of OHCs was more sever. Possible reasons of different fates IHCs and OHCs in our study might include the following. Previous study reported that the cortical network involved in the sound-induced electromotility of OHCs contains SPTAN1 [18], and we speculated that the depletion of Sptan1 may decrease the electromotility of OHCs. In addition, we cannot definitively exclude the presence of unknown partners, exclusively expressed in IHCs and OHCs that modulate Sptanl or undergo other regulations. A difference in actin or microtubule metabolism in OHCs versus IHCs might also explain the difference of phenotype between the two HCs. Furthermore, Sptan1 is also involved in diverse processes occurring in the nucleus, and the loss of Sptanl in HCs could have led to chromosome instability [49]. Sptanl is speculated to be a major substrate for calpain and caspase-3 cysteine proteases [50]. In addition, Sptan1-CKO mice may also undergo necroptosis and necrosis, but we did not detect them. Therefore, the cell death-related pathway affected after Sptanl knockout remains to be determined.

In conclusion, our findings suggested that the Sptan1CKO mice displayed abnormal OHC and IHC stereocilia morphology, including improper bundle orientation, and the cuticular plate lacked its characteristic oval shape, which was more pronounced in OHCs. After hearing onset (P12), the $\mathrm{OHC}$ stereocilia showed rapid degeneration, followed by HCs loss proceeding from apex to base. Sptan1 depletion may affect the formation of integrin-focal adhesions, leading to abnormal actin cytoskeletal structure and decreased cell spreading. Therefore, our study indicated that Sptan1 is essential for the cochlear hair cell morphology and function, which plays the role via the regulation of focal adhesion signaling.

Supplementary Information The online version contains supplementary material available at https://doi.org/10.1007/s12035-021-02551-2.

Acknowledgements We would like to thank Prof. Dalian Ding and Prof. Jian Wang for the experimental guidance and useful discussion of the work. We are grateful to Prof. Renjie Chai for providing the Gfil-cre mice, previously obtained from Prof. Lin Gan.

Author Contribution All authors reviewed the report and approved the final version. DY, FL, and SY designed all experiments. QY performed all of the animal experiments except auditory electrophysiological recordings, which were performed by $\mathrm{HC}$. QY and $\mathrm{HW}$ performed the in vitro experiments. ZL and YJ acquired and analyzed some data. ZL, JW, and YX helped with the experimental design and data interpretation. We assigned the authorship order according to the amount of work carried out by each author.

Funding This work was supported by the National Natural Science Foundation of China (No. 81870717, 81971240, 81900936), and the International Cooperation and Exchange of the National Natural Science Foundation of China (No. 81720108010). The funder had no role in study design, data collection, or analysis or in the presentation or publication of the results. 
Data Availability The datasets used and/or analyzed during the current study are available from the corresponding author on reasonable request.

\section{Declarations}

Ethics Approval We performed all animal procedures according to protocols that were approved by the Animal Care and Use Committee of Shanghai Jiao Tong University Affiliated Sixth People's Hospital and that were consistent with the National Institute of Health's Guide for the Care and Use of Laboratory Animals.

Consent for Publication All authors consent to the publication of current data.

Consent to Participate Not applicable.

Competing Interests The authors declare no competing interests.

Open Access This article is licensed under a Creative Commons Attribution 4.0 International License, which permits use, sharing, adaptation, distribution and reproduction in any medium or format, as long as you give appropriate credit to the original author(s) and the source, provide a link to the Creative Commons licence, and indicate if changes were made. The images or other third party material in this article are included in the article's Creative Commons licence, unless indicated otherwise in a credit line to the material. If material is not included in the article's Creative Commons licence and your intended use is not permitted by statutory regulation or exceeds the permitted use, you will need to obtain permission directly from the copyright holder. To view a copy of this licence, visit http://creativecommons.org/licenses/by/4.0/.

\section{References}

1. Akbariazar E, Vahabi A, Abdi Rad I (2019) Report of a novel splicing mutation in the myo15a gene in a patient with sensorineural hearing loss and spectrum of the myo15a mutations. Clin Med Insights Case Rep 12:1179547619871907. https://doi.org/ 10.1177/1179547619871907

2. Forge A, Taylor R, Dawson S, Lovett M, Jagger D (2017) Disruption of sorcs 2 reveals differences in the regulation of stereociliary bundle formation between hair cell types in the inner ear. PLoS Genet 13:e1006692

3. Barr-Gillespie PG (2015) Assembly of hair bundles, an amazing problem for cell biology. Mol Biol Cell 26:2727-2732. https://doi. org/10.1091/mbc.E14-04-0940

4. Diaz-Horta O, Subasioglu-Uzak A, Grati M, DeSmidt A, Foster J, Cao L, Bademci G, Tokgoz-Yilmaz S et al (2014) Fam65b is a membrane-associated protein of hair cell stereocilia required for hearing. Proc Natl Acad Sci USA 111:9864-9868

5. Flock A, Cheung HC (1977) Actin filaments in sensory hairs of inner ear receptor cells. J Cell Biol 75:339-343

6. Tilney L, Derosier D, Mulroy M (1980) The organization of actin filaments in the stereocilia of cochlear hair cells. J Cell Biol $86: 244-259$

7. Delprat B, Michel V, Goodyear R, Yamasaki Y, Michalski N, El-Amraoui A, Perfettini I, Legrain P et al (2005) Myosin xva and whirlin, two deafness gene products required for hair bundle growth, are located at the stereocilia tips and interact directly. Hum Mol Genet 14:401-410. https://doi.org/10.1093/hmg/ddi036
8. Park SN, Back SA, Park KH, Kim DK, Park SY, Oh JH, Park YS, Yeo SW (2010) Comparison of cochlear morphology and apoptosis in mouse models of presbycusis. Clin Exp Otorhinolaryngol 3:126-135. https://doi.org/10.3342/ceo.2010.3.3.126

9. Pollock LM, McDermott BM Jr (2015) The cuticular plate: a riddle, wrapped in a mystery, inside a hair cell. Birth Defects Res C Embryo Today 105:126-139

10. Holley MC, Ashmore JF (1990) Spectrin, actin and the structure of the cortical lattice in mammalian cochlear outer hair cells. J Cell Sci 96(Pt 2):283-291

11. Mahendrasingam S, Furness DN, Hackney CM (1998) Ultrastructural localisation of spectrin in sensory and supporting cells of guinea-pig organ of corti. Hear Res 126:151-160

12. Seiler C, Ben-David O, Sidi S, Hendrich O, Rusch A, Burnside B, Avraham KB, Nicolson T (2004) Myosin vi is required for structural integrity of the apical surface of sensory hair cells in zebrafish. Dev Biol 272:328-338. https://doi.org/10.1016/j.ydbio. 2004.05.004

13. Drummond MC, Belyantseva IA, Friderici KH, Friedman TB (2012) Actin in hair cells and hearing loss. Hear Res 288:89-99. https://doi.org/10.1016/j.heares.2011.12.003

14. Perrin B, Sonnemann K, Ervasti J (2010) B-actin and $\gamma$-actin are each dispensable for auditory hair cell development but required for stereocilia maintenance. PLoS Genet 6:e1001158. https://doi. org/10.1371/journal.pgen.1001158

15. Seki Y, Miyasaka Y, Suzuki S, Wada K, Yasuda S, Matsuoka K, Ohshiba Y, Endo K et al (2017) A novel splice site mutation of myosin vi in mice leads to stereociliary fusion caused by disruption of actin networks in the apical region of inner ear hair cells. PLoS ONE 12:e0183477. https://doi.org/10.1371/journal.pone. 0183477

16. Hertzano R, Shalit E, Rzadzinska A, Dror A, Song L, Ron U, Tan J, Shitrit A et al (2008) A myo6 mutation destroys coordination between the myosin heads, revealing new functions of myosin vi in the stereocilia of mammalian inner ear hair cells. PLoS Genet 4:e1000207. https://doi.org/10.1371/journal.pgen.1000207

17. Yao Q, Wang H, Li Z, Yu D, Yin S (2019) Preliminary study on screening cochlear proteins interacting with myosin vi. J Otolaryngol Ophthalmol Shandong Univ 33:40-43

18. Legendre K, Safieddine S, Kussel-Andermann P, Petit C, ElAmraoui A (2008) Alphaii-betav spectrin bridges the plasma membrane and cortical lattice in the lateral wall of the auditory outer hair cells. J Cell Sci 121:3347-3356. https://doi.org/10. $1242 /$ jcs. 028134

19. Ackermann A, Brieger A (2019) Athe role of nonerythroid spectrin ii in cancer. Journal of oncology 2019:7079604. https://doi. org/10.1155/2019/7079604

20. Huang C, Zhang C, Ho T, Oses-Prieto J, Burlingame A, Lalonde J, Noebels J, Leterrier C et al (2017) Aii spectrin forms a periodic cytoskeleton at the axon initial segment and is required for nervous system function. J Neurosci 37:11311-11322

21. Liu Y, Qi J, Chen X, Tang M, Chu C, Zhu W, Li H, Tian C et al (2019) Critical role of spectrin in hearing development and deafness. Sci Adv 5:eaa7803. https://doi.org/10.1126/sciadv.aav7803

22. Yang H, Wang H, Shivalila C, Cheng A, Shi L, Jaenisch R (2013) One-step generation of mice carrying reporter and conditional alleles by crispr/cas-mediated genome engineering. Cell 154:1370-1379

23. Cong L, Ran F, Cox D, Lin S, Barretto R, Habib N, Hsu P, Wu $\mathrm{X}$ et al (2013) Multiplex genome engineering using crispr/cas systems. Science 339:819-823

24. Yang H, Gan J, Xie X, Deng M, Feng L, Chen X, Gao Z, Gan L (2010) Gfil-cre knock-in mouse line: a tool for inner ear hair cellspecific gene deletion. Genesis (New York N.Y 2000) 48:400406. https://doi.org/10.1002/dvg.20632 
25. Seliger H (1990) Pcr protocols — a guide to methods and applications. Trends Biotechnol 8:335-335

26. Jing Z, Rutherford M, Takago H, Frank T, Fejtova A, Khimich D, Moser T, Strenzke N (2013) Disruption of the presynaptic cytomatrix protein bassoon degrades ribbon anchorage, multiquantal release, and sound encoding at the hair cell afferent synapse. J Neurosci 33:4456-4467

27. Neef J, Gehrt A, Bulankina A, Meyer A, Riedel D, Gregg R, Strenzke N, Moser T (2009) The ca2+ channel subunit beta2 regulates ca2+ channel abundance and function in inner hair cells and is required for hearing. J Neurosci 29:10730-10740

28. Ding DL, Wang J, Salvi R, Henderson D, Hu BH, McFadden SL, Mueller M (1999) Selective loss of inner hair cells and type-i ganglion neurons in carboplatin-treated chinchillas. Mechanisms of damage and protection. Ann N Y Acad Sci 884:152-170

29. Zhang J, Sun H, Salvi R, Ding D (2018) Paraquat initially damages cochlear support cells leading to anoikis-like hair cell death. Hear Res 364:129-141. https://doi.org/10.1016/j.heares.2018.03. 014 (S0378-5955(17)30618-4 [pii])

30. Livak KJ, Schmittgen TD (2001) Analysis of relative gene expression data using real-time quantitative pcr and the $2-\delta \delta$ $\mathrm{c} t$ method. Methods-A Companion To Methods in Enzymology 25:402-408

31. May-Simera, H (2016) Evaluation of planar-cell-polarity phenotypes in ciliopathy mouse mutant cochlea. J Vis Exp 53559. https://doi.org/10.3791/53559.

32. Anniko M (1983) Cytodifferentiation of cochlear hair cells. Am J Otolaryngol 4:375-388. https://doi.org/10.1016/s0196-0709(83) 80043-x

33. Kitajiri S, Sakamoto T, Belyantseva IA, Goodyear RJ, Stepanyan R, Fujiwara I, Bird JE, Riazuddin S et al (2010) Actin-bundling protein triobp forms resilient rootlets of hair cell stereocilia essential for hearing. Cell 141:786-798. https://doi.org/10.1016/j.cell. 2010.03.049

34. Stankewich M, Cianci C, Stabach P, Ji L, Nath A, Morrow J (2011) Cell organization, growth, and neural and cardiac development require $\alpha$ ii-spectrin. J Cell Sci 124:3956-3966. https:// doi.org/10.1242/jcs.080374

35. Savoy-Burke G, Gilels F, Pan W, Pratt D, Que J, Gan L, White P, Kiernan A (2014) Activated notch causes deafness by promoting a supporting cell phenotype in developing auditory hair cells. PLoS ONE 9:e108160. https://doi.org/10.1371/journal.pone.0108160

36. Yu DZ, Ding DL, Yin SK, Salvi RJ (2009) Ototoxic effects of streptomycin in vestibular organotypic cultures. Zhonghua Er Bi Yan Hou Tou Jing Wai Ke Za Zhi 44:53-56

37. Hu BH, Henderson D, Nicotera TM (2006) Extremely rapid induction of outer hair cell apoptosis in the chinchilla cochlea following exposure to impulse noise. Hear Res 211:16-25. https://doi.org/ 10.1016/j.heares.2005.08.006

38. Kelly G, Faraj R, Zhang Y, Maltepe E, Fineman J, Black S, Wang $\mathrm{T}$ (2019) Pulmonary endothelial mechanical sensing and signaling, a story of focal adhesions and integrins in ventilator induced lung injury. Front Physiol 10:511. https://doi.org/10.3389/fphys. 2019.00511

39. Khan R, Goult B (2019) Adhesions assemble!-autoinhibition as a major regulatory mechanism of integrin-mediated adhesion. Front Mol Biosci 6:144. https://doi.org/10.3389/fmolb.2019.00144

40. Littlewood Evans A, Müller U (2000) Stereocilia defects in the sensory hair cells of the inner ear in mice deficient in integrin alpha8beta1. Nat Genet 24:424-428. https://doi.org/10.1038/ 74286

41. Goodman L, Zallocchi M (2017) Integrin $\alpha 8$ and pcdh15 act as a complex to regulate cilia biogenesis in sensory cells. J Cell Sci 130:3698-3712. https://doi.org/10.1242/jcs.206201

42. Schoenwaelder S, Burridge K (1999) Bidirectional signaling between the cytoskeleton and integrins. Curr Opin Cell Biol 11:274-286. https://doi.org/10.1016/s0955-0674(99)80037-4

43. Müller U, Littlewood-Evans A (2001) Mechanisms that regulate mechanosensory hair cell differentiation. Trends Cell Biol 11:334-342. https://doi.org/10.1016/s0962-8924(01)02046-3

44. Jamesdaniel S, Hu B, Kermany M, Jiang H, Ding D, Coling D, Salvi R (2011) Noise induced changes in the expression of p38/ mapk signaling proteins in the sensory epithelium of the inner ear. J Proteomics 75:410-424. https://doi.org/10.1016/j.jprot.2011.08. 007

45. Henson M, Burridge K, Fitzpatrick D, Jenkins D, Pillsbury H, Henson O (1985) Immunocytochemical localization of contractile and contraction associated proteins in the spiral ligament of the cochlea. Hear Res 20:207-214. https://doi.org/10.1016/03785955(85)90025-5

46. Surel, C, Guillet, M, Lenoir, M, Bourien, J, Sendin, G, Joly, W, Delprat, B, Lesperance, M, Puel, J \& Nouvian, R (2016) Remodeling of the inner hair cell microtubule meshwork in a mouse model of auditory neuropathy auna1. eNeuro, 3. https://doi.org/ 10.1523/eneuro.0295-16.2016

47. Schacht J, Talaska A, Rybak L (2012) Cisplatin and aminoglycoside antibiotics: hearing loss and its prevention. Anat Rec (Hoboken N.J 2007) 295:1837-1850. https://doi.org/10.1002/ar.22578

48. Saunders J, Dear S, Schneider M (1985) The anatomical consequences of acoustic injury: a review and tutorial. J Acoust Soc Am 78:833-860. https://doi.org/10.1121/1.392915

49. Lambert MW (2015) Functional significance of nuclear alpha spectrin. J Cell Biochem 116:1816-1830. https://doi.org/10.1002/ jcb. 25123

50. Cardali S, Maugeri R (2006) Detection of alphaii-spectrin and breakdown products in humans after severe traumatic brain injury. J Neurosurg Sci 50:25-31

Publisher's Note Springer Nature remains neutral with regard to jurisdictional claims in published maps and institutional affiliations. 